\title{
Circulating markers of NADH-reductive stress correlate with mitochondrial disease severity
}

\author{
Rohit Sharma, ${ }^{1,2,3,4}$ Bryn Reinstadler, ${ }^{1,2,3,4}$ Kristin Engelstad, ${ }^{5}$ Owen S. Skinner, ${ }^{1,2,3,4}$ Erin Stackowitz, ${ }^{5}$ Ronald G. Haller, ${ }^{6,7}$ \\ Clary B. Clish, ${ }^{4}$ Kerry Pierce, ${ }^{4}$ Melissa A. Walker, ${ }^{1,2,3,4,8}$ Robert Fryer, ${ }^{5}$ Devin Oglesbee, ${ }^{9}$ Xiangling Mao, ${ }^{10}$ Dikoma C. Shungu, ${ }^{10}$ \\ Ashok Khatri, ${ }^{11}$ Michio Hirano, ${ }^{5}$ Darryl C. De Vivo, ${ }^{5}$ and Vamsi K. Mootha ${ }^{1,2,3,4}$ \\ ${ }^{1}$ Howard Hughes Medical Institute, Department of Molecular Biology, and ${ }^{2}$ Department of Medicine, Massachusetts General Hospital, Boston, Massachusetts, USA. ${ }^{3}$ Department of Systems Biology, Harvard \\ Medical School, Boston, Massachusetts, USA. ${ }^{4}$ Broad Institute, Cambridge, Massachusetts, USA. ${ }^{5}$ Department of Neurology, Columbia University Irving Medical Center, New York, New York, USA. ${ }^{6}$ Department \\ of Neurology and Neurotherapeutics, University of Texas Southwestern Medical Center, Dallas, Texas, USA. 'Institute for Exercise and Environmental Medicine of Texas Health Presbyterian Hospital, Dallas, \\ Texas, USA. ${ }^{8}$ Department of Neurology, Massachusetts General Hospital, Boston, Massachusetts, USA. ${ }^{9}$ Department of Laboratory Medicine and Pathology, Mayo Clinic, Rochester, Minnesota, USA. \\ ${ }^{10}$ Department of Radiology, Weill Cornell Medicine, New York, New York, USA. "Endocrine Division and Department of Medicine, Massachusetts Ceneral Hospital, Boston, Massachusetts, USA.
}

\begin{abstract}
Mitochondrial disorders represent a large collection of rare syndromes that are difficult to manage both because we do not fully understand biochemical pathogenesis and because we currently lack facile markers of severity. The m.3243A $>C$ variant is the most common heteroplasmic mitochondrial DNA mutation and underlies a spectrum of diseases, notably mitochondrial encephalomyopathy lactic acidosis and stroke-like episodes (MELAS). To identify robust circulating markers of m.3243A $>C$ disease, we first performed discovery proteomics, targeted metabolomics, and untargeted metabolomics on plasma from a deeply phenotyped cohort (102 patients, 32 controls). In a validation phase, we measured concentrations of prioritized metabolites in an independent cohort using distinct methods. We validated 20 analytes (1 protein, 19 metabolites) that distinguish patients with MELAS from controls. The collection includes classic (lactate, alanine) and more recently identified (CDF-15, $\alpha$-hydroxybutyrate) mitochondrial markers. By mining untargeted mass-spectra we uncovered 3 less well-studied metabolite families: $\mathrm{N}$-lactoyl-amino acids, $\boldsymbol{\beta}$-hydroxy acylcarnitines, and $\boldsymbol{\beta}$-hydroxy fatty acids. Many of these $\mathbf{2 0}$ analytes correlate strongly with established measures of severity, including Karnofsky status, and mechanistically, nearly all markers are attributable to an elevated NADH/NAD+ ratio, or NADH-reductive stress. Our work defines a panel of organelle function tests related to NADH-reductive stress that should enable classification and monitoring of mitochondrial disease.
\end{abstract}

\section{Introduction}

With the initial detection of the mitochondrial respiratory chain, it was anticipated that its dysfunction would lead to metabolic perturbations and disease (1). There is now widespread appreciation that variants in either the nuclear or mitochondrial DNA (mtDNA) lead to respiratory chain dysfunction and disease. Collectively, respiratory chain diseases comprise a common inborn error of metabolism with an estimated prevalence of at least 1 in 4300, manifesting with marked phenotypic heterogeneity, striking at any age, and involving any organ system (2-4). Quantitative declines in respiratory chain activities are also observed in many

\section{Related Commentary: https://doi.org/10.1172/JCI145158}

\footnotetext{
Conflict of interest: VKM is on the scientific advisory board and receives equity from Janssen Pharmaceuticals and 5AM Ventures. VKM is an inventor on a patent application filed by Massachusetts General Hospital on novel therapeutic approaches targeting reductive stress ("Extracellular Redox Enzyme System to Alleviate Disease," no. 16/769,319). OSS is a paid consultant for Proteinaceous Inc. MH is a paid consultant for Zogenix and Entrada Therapeutics. DCDV is a paid consultant for Biogen and AveXis Therapeutics. RS holds equity in BlueBird Bio.

Copyright: (? 2021, American Society for Clinical Investigation.

Submitted: December 30, 2019; Accepted: November 18, 2020 ;

Published: January 19, 2021

Reference information: J Clin Invest. 2021;131(2):e136055.

https://doi.org/10.1172/JCl136055.
}

common diseases, such as diabetes, Parkinson's disease, and sarcopenia, as well as the aging process itself (5-9).

One of the most frequent causes of inherited mitochondrial disease is the heteroplasmic m.3243A $>$ G mutation in the MT-TL1 gene encoding the mitochondrial leucyl-tRNA 1 gene (10-12). This variant is responsible for nearly $80 \%$ of cases of mitochondrial encephalomyopathy lactic acidosis and strokelike episodes (MELAS), one of the most common mtDNA disorders, which typically presents before age 40 years with myopathy, headaches, vomiting, hearing loss, seizures, lactic acidosis, and stroke-like episodes $(10,13)$. MELAS typically progresses relentlessly and unpredictably with the average survival from the first stroke-like episode being 16.9 years (14). Patients with MELAS are typically afflicted with a host of other comorbidities including growth deficits, diabetes, and cardiomyopathy. Most patients with the $\mathrm{m} .3243 \mathrm{~A}>\mathrm{G}$ mutation do not manifest the full MELAS spectrum, but rather milder phenotypes, including maternally inherited diabetes and deafness (MIDD) or chronic progressive external ophthalmoplegia (CPEO). This marked phenotypic heterogeneity is attributed to the combined influences of tissue mtDNA mutation load (heteroplasmy) and differential tissue sensitivity to defective respiratory chain function observed in all mitochondrial disorders. The prevalence of the m.3243A $>\mathrm{G}$ variant is as high as approximately 1 in 400 and the prevalence of MELAS is estimated at 0.2 in 100,000 adults 


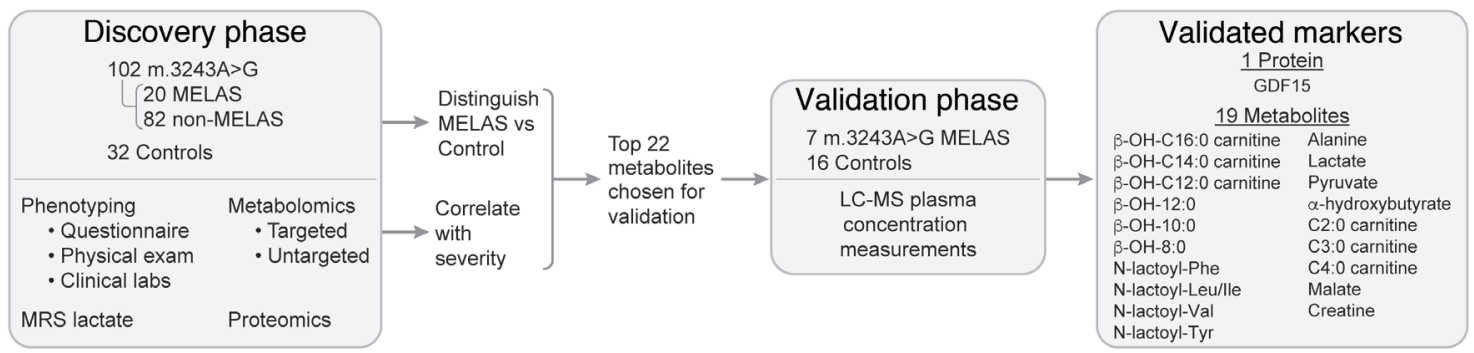

Figure 1. MELAS biomarker discovery and validation. Over a 3-year period, a discovery cohort of control patients harboring the m.3243A $>\mathrm{G}$ variant was phenotyped through a combination of questionnaires, physical exams, and clinical laboratory measurements. Plasma metabolomic and proteomic profiles of the discovery cohort were analyzed to identify analytes that best distinguished patients with MELAS from controls, and that most strongly correlated with disease severity. From these, 22 metabolites were chosen for validation in an independent cohort of 7 patients with m.3243A>C MELAS and 16 controls. This pipeline yielded a validated set of 20 plasma markers (1 protein and 19 metabolites).

(3, 15-18). There are no proven MELAS treatments, though several therapies have indicated anecdotal benefit and clinical trials are underway $(19,20)$.

While the molecular genetic diagnoses of mitochondrial disorders has been transformed by next-generation DNA sequencing, clinical management continues to be hindered by an incomplete understanding of their biochemical pathogenesis and also by the lack of facile, quantitative biomarkers of disease progression (21, 22). Biochemical testing of patients with MELAS shows respiratory chain dysfunction, often in complex I, and a backlog of NADH-reducing equivalents $(23,24)$ causing an elevated NADH/ $\mathrm{NAD}^{+}$ratio, known as NADH-reductive stress, that drives elevation of coupled metabolites such as lactate (24-31). While heteroplasmy levels in blood can correlate with severity, these levels actually gradually decrease over time while the disease progresses (32-36). Brain lactate levels, as measured by proton magnetic resonance spectroscopy (MRS), strongly discriminate patients from controls, and correlate with severity (37). Recent metabolomics studies of patients with and mouse models of mitochondrial diseases have nominated additional markers that await validation $(38,39)$. A large, focused study of one genetically monomorphic respiratory chain disease would help establish a robust biochemical signature for mitochondrial dysfunction with potential implications for rare and common disorders.

Here, we report a biomarker discovery effort for MELAS that comprised a 2-stage approach involving discovery and validation (Figure 1). First, together with phenotypic information, brain MRS, and urine heteroplasmy, we collected targeted and untargeted plasma metabolomics and proteomics measurements for a discovery cohort of 102 patients with the m.3243A $>$ G variant. Using these data, we prioritized a set of proteins and metabolites that strongly distinguish patients with MELAS from controls. In the validation stage, we used an independently collected m.3243A $>$ G cohort and independent, quantitative analytical methods to validate 1 protein and 19 metabolites distinguishing patients with MELAS from controls (Figure 1). The biochemical signature includes classic markers, recently identified protein and metabolite markers, and a set of new analytes, such as $\mathrm{N}$ lactoyl-amino acids, never linked to mitochondrial dysfunction. Importantly, many analytes in our panel quantitatively track with disease severity and underscore NADH redox imbalance as a predominant feature of mitochondrial respiratory chain disease.

\section{Results}

Phenotypic, laboratory, and MRS characterization of the m.3243A>G discovery cohort. With the ultimate goals of using metabolomic and proteomic measurements to both discriminate patients with MELAS from controls as well as to correlate with disease severity, we documented histories, performed exams, collected blood and urine samples, and performed MRS on a discovery cohort of 134 individuals (Figure 1). Twenty individuals harboring the m.3243A $>\mathrm{G}$ variant had a documented stroke-like event and fulfilled conservative clinical criteria to comprise the MELAS cohort (Table 1, Supplemental Table 1; supplemental material available online with this article; https://doi.org/10.1172/JCI136055DS1) (10). The patient cohort also consisted of 82 individuals with $\mathrm{m} .3243 \mathrm{~A}>\mathrm{G}$ mutations (harboring the variant or who were obligate carriers) with a range of disease features but without stroke-like episode who we refer to here as m.3243A $>$ G non-MELAS patients. All individuals, including the 32 controls, were in their baseline state of health at the time of participation and their clinic visits were coordinated to complete all aspects of the study within 2 days (Supplemental Table 1). All samples were first morning samples and the majority of participants were fasting except for a small fraction $(<10 \%)$ who required food with their morning medications, which were taken at least 2 hours prior to sample collection (Table 2 and Methods).

Comparisons of the clinical features across the 3 groups were consistent with previous natural history studies demonstrating severe deficits across multiple systems with m.3243A $>G$ nonMELAS patients being intermediate between controls and patients with MELAS (Table 1) (40). As seen in prior studies, patients with MELAS had a lower mean body mass index (BMI) (Table 1) (40). The majority of patients with MELAS showed deficits in their neurological exams (Columbia Neurological Score), cognitive abilities (Folstein Mini-Mental State Examination Score), and memory. Muscle strength and 6-minute walk test, both reflections of myopathy, were substantially impaired in patients with MELAS. Hearing loss $(80 \%)$, diabetes $(60 \%)$, and gastrointestinal complaints $(75 \%)$ were frequent MELAS complications. These multisystemic deficits were associated with markedly worse Karnofsky performance status scores in both patients with MELAS and m.3243A $>\mathrm{G}$ non-MELAS patients as compared with controls. The wide spectrum of severities was evident in $\mathrm{m} .3243 \mathrm{~A}>\mathrm{G}$ non-MELAS patients, as several displayed lower Karnofsky scores than the highest scoring patients with MELAS. 
Table 1. Demographics and disease features of study participants

\begin{tabular}{|c|c|c|c|c|}
\hline & \multirow{2}{*}{\multicolumn{3}{|c|}{ Discovery cohort }} & \multirow{4}{*}{ Units (range) } \\
\hline & & & & \\
\hline & \multicolumn{2}{|c|}{$\mathrm{m} .3243 \mathrm{~A}>\mathrm{G}$} & \multirow[b]{2}{*}{ Controls } & \\
\hline & m.3243A>C MELAS (+ strokes) & m.3243A>C non-MELAS (- strokes) & & \\
\hline$N$ & 20 & 82 & 32 & \\
\hline Age, mean (range) & $38.2(5-69)$ & $39.3(4-68)$ & $43.8(20-83)$ & years \\
\hline BMI in adults, mean (range) & $22.9(15.3-37.3)$ & $24.8(15.2-40.7)$ & $26.8(17.4-35.7)$ & $\mathrm{kg} / \mathrm{m}^{2}$ \\
\hline \multicolumn{5}{|l|}{ Cardinal features } \\
\hline Stroke-like episodes ${ }^{A}$ & $20(100)$ & $0(0)$ & $0(0)$ & number $(\%)$ \\
\hline Columbia neurological score ${ }^{B}$ & $61.8(8.8)^{* * *}$ & $70.1(6.0)^{* *}$ & $73.3(3.3)$ & score (SD) [0-76] \\
\hline Karnofsky score ${ }^{\mathrm{C}}$ & $62.5(13.4)^{* * *}$ & $86.7(9.9)^{* * *}$ & $96.6(4.8)$ & score (SD) [0-100] \\
\hline Folstein mini-mental state examination score ${ }^{D}$ & $24.9(6.1)^{* *}$ & $28.4(1.7)$ & $29.0(1.3)$ & score (SD) [0-30] \\
\hline Neuropathy & $10(50)^{* *}$ & $18(22)$ & $3(9.4)$ & number $(\%)$ \\
\hline Psychiatric condition & $12(60)^{* *}$ & $32(39)$ & $6(18.8)$ & number $(\%)$ \\
\hline Memory problems ${ }^{\complement}$ & $-2.22(1.8)^{*}$ & $-0.5(0.9)$ & $-0.7(0.9)$ & z score (SD) \\
\hline \multicolumn{5}{|l|}{ Visceral } \\
\hline Exercise intolerance & $15(75)^{* * *}$ & $30(58.8)^{* * *}$ & $0(0)$ & number (\%) \\
\hline Six-minute walk & $67.7(14.7)^{* *}$ & $86.7(18.7)$ & $84.6(19.7)$ & \% predicted (SD) \\
\hline Hearing loss & $16(80)^{* * *}$ & $31(38)^{* *}$ & $3(9)$ & number (\%) \\
\hline Short stature ${ }^{H}$ & $3(15)^{* *}$ & $0(0)$ & $0(0)$ & number $(\%)$ \\
\hline Growth failure ${ }^{H}$ & $1(5)$ & $1(1.3)$ & $0(0)$ & number $(\%)$ \\
\hline Diabetes & $12(60)^{* * *}$ & $22(27)^{*}$ & $2(6)$ & number (\%) \\
\hline Cardiomyopathy & $4(20)^{* *}$ & $2(2)$ & $0(0)$ & number $(\%)$ \\
\hline
\end{tabular}

Laboratory studies of the discovery cohort revealed expected disease-associated differences (Table 2). Urine heteroplasmy was progressively higher from controls to m.3243A $>\mathrm{G}$ nonMELAS patients to patients with MELAS, albeit with wide variation, consistent with prior studies $(36,41)$. MRS ventricular lactate, a recognized marker of MELAS severity (37), was found to be progressively higher in the patient groups. As a corroboration of urine heteroplasmy and MRS lactate as readouts of severity, we observed moderately strong correlations of each measure with the Karnofsky score ( $\tau=-0.49$ and -0.42 , respectively) $(41,42)$.

Although standard blood cell counts, electrolytes, liver function tests, and endocrine parameters were formally within the normal reference ranges, patients with MELAS and controls showed marked differences in certain laboratory values (Table 2). Patients with MELAS had lower red blood cell counts but higher total white blood cell counts, although their subtypes showed no significant differences (data not shown). An elevated corrected anion gap in patients with MELAS likely reflected a mild baseline metabolic acidosis. Alkaline phosphatase, globulin, and lactate dehydrogenase (LDH) were also substantially increased in MELAS. As previously observed, total triiodothyronine (T3) is markedly lower in patients with MELAS (40). Finally, elevated hemoglobin A1c levels were consistent with the impaired glucose tolerance associated with MELAS (43). In summary, clinical characterization of the discovery cohort recapitulated the multisystemic nature of MELAS and highlighted MRS ventricular lactate and urine heteroplasmy as quantitative markers of functional severity $(37,40)$.

Proteomics validated GDF-15 and nominated sE-selectin, HS6ST1, and RET as candidate biomarkers. To identify protein markers of MELAS, we performed proteomic profiling on plasma samples from $16 \mathrm{~m} .3243 \mathrm{~A}>\mathrm{G}$ patients with MELAS, 60 m.3243A $>$ G non-MELAS patients (i.e., those without stroke-like 
Table 2. Clinical laboratory values of study participants

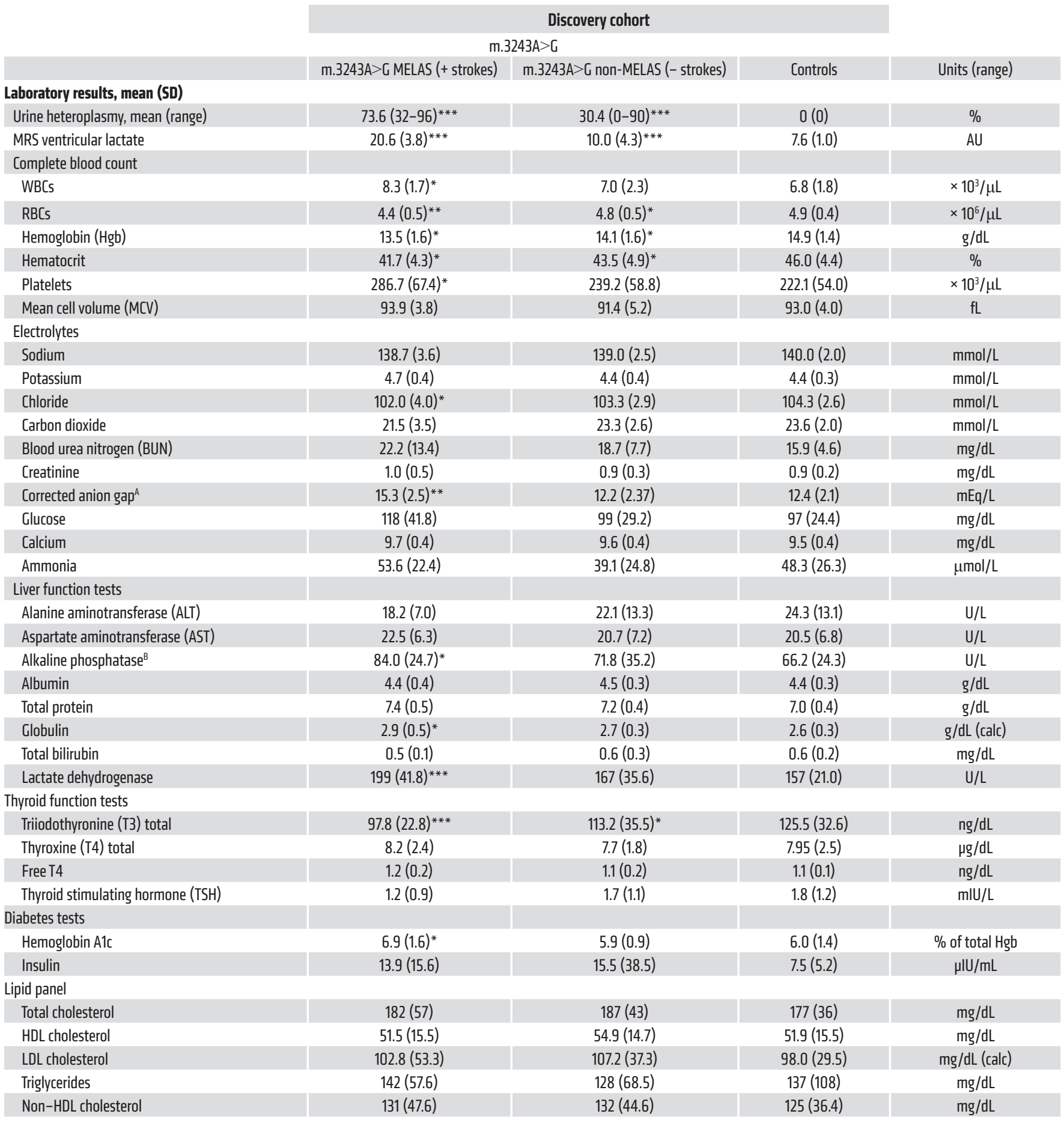

Patient information was gathered at the time of sample collection. See Table 1 for patient demographics. Laboratory data show mean and standard deviation except where noted for controls $(n=32)$, m.3243A>C non-MELAS (- strokes) $(n=76)$, and m.3243A>C MELAS (+ strokes) ( $n=13)$. Wilcoxon ranksum $P$ values comparing patient groups compared with controls are indicated by asterisks as follows: ${ }^{*} P<0.05,{ }^{* *} P<0.01$, and ${ }^{* * *} P<0.001$. ${ }^{A} T$ he anion gap is corrected for the albumin concentration: $\left([\mathrm{Na}]-\left([\mathrm{Cl}]+\left[\mathrm{HCO}_{3}\right]\right)+2.5 \times\left(4 \mathrm{~g} / \mathrm{dL}-[\right.\right.$ albumin] $) .{ }^{\mathrm{B}}$ Alkaline phosphatase values here only include adults. 
episodes), and 24 controls using an aptamer-based multiplexed assay (SOMAscan, SomaLogic Inc.) that quantifies 1310 proteins (Supplemental Table 1). Since the proteome and metabolome are known to change with age, sex, and BMI, we compared levels of each protein in MELAS and controls after controlling for these variables and identified 4 proteins meeting a false discovery rate (FDR) threshold of 2\% (Figure 2A and Supplemental Figure 1A). The most markedly changed among these was growth differentiation factor 15 (GDF-15), which validated previous studies that found it to be elevated in mitochondrial disease, including MELAS (Figure 2, A and B) (44-46). Also identified as substantially altered were the levels of 3 plasma proteins. Compared with controls, patients with MELAS showed increased levels of sE-selectin but modestly decreased levels of heparan sulfate 6-O-sulfotransferase 1 (HS6ST1) and receptor tyrosine kinase (RET) (Figure 2, C-E). In summary, proteomic profiling of the discovery cohort provided formal validation of GDF-15 as a MELAS marker while nominating 3 new protein markers.

Metabolomics analyses recovered classic markers while nominating new ones. We applied 4 complementary liquid chromatographymass spectrometry (LC-MS) methods (Broad Institute) to measure relative levels of 376 identified polar and nonpolar metabolites in 134 plasma samples (20 m.3243A $>$ G patients with MELAS, 82 m.3243A $>$ G non-MELAS patients, 32 controls). After controlling for age, sex, and BMI and applying a 2\% FDR cutoff, we identified 23 metabolites in this discovery cohort that were significantly different between patients with MELAS and controls (Figure 2F, and Supplemental Figure 1B). Three classic respiratory chain disease markers (lactate, pyruvate, and alanine) were prominent among the top targeted metabolites distinguishing patients with MELAS from controls and, along with GDF-15 above, validated our discovery pipeline (Figure 2, $\mathrm{F}$ and $\mathrm{G})(47,48)$.

Other metabolites highlighted in this analysis included familiar as well as recently nominated markers. Elevations of tricarboxylic acid (TCA) cycle intermediates have been reported in mitochondrial diseases and, indeed, we found patients with MELAS have small but significant increases in citrate, aconitate, isocitrate, and malate (Figure $2 \mathrm{H}$ ) (48). However, not all TCA intermediates were increased, as succinate was actually reduced, barely missing our significance threshold (Supplemental Figure 2A).

The suite of top metabolites included several that feed into the TCA cycle. C2:0-carnitine, a product of acetyl-CoA, into which pyruvate and fatty acid $\beta$-oxidation funnel, was higher in patients with MELAS (Figure 2H). Similarly, C3:0-carnitine is a product of propionyl-CoA, into which catabolic products of odd chain fatty acids and several amino acids pass en route to the TCA cycle and which is also elevated in patients with MELAS (Figure $2 \mathrm{H}$ ). Furthermore, in analogous biochemical organization to pyruvate and lactate, $\alpha$-ketobutyrate can be converted to C3:0-carnitine or reduced to $\alpha$-hydroxybutyrate by LDH. Interestingly, $\alpha$-hydroxybutyrate was increased in patients with MELAS (Figure 2I), confirming studies that identified this metabolite as a robust marker of mitochondrial disease as well as hepatic $\mathrm{NADH} / \mathrm{NAD}^{+}$ratio in mouse studies (49-52).

Acylcarnitines are known to be elevated in mitochondrial disorders and comprise 8 of the top 23 metabolites (Figure 2, F and J). Targeted metabolomics reported 26 acylcarnitines species and indicated the MELAS pattern includes short chain acylcarnitines with less than 5 carbons as well as the 3 hydroxy-acylcarnitines species detected (Figure 2K). However, as these were the only 3 hydroxy-acylcarnitines reported, a complete MELAS acylcarnitine signature could not be resolved from this platform. Carnitine itself as well as creatine, both previously nominated mitochondrial disease markers, were markedly increased in patients with MELAS (Figure 2L) (53). The 4 patients with MELAS taking carnitine and 2 taking a cocktail of mitochondrial vitamins (none were taking creatine) did not account for these or the acylcarnitine observations. Although all but 4 patients with MELAS were taking supplements, the number taking any individual supplement was too small to rigorously determine correlations with any metabolite. In summary, targeted metabolomic profiling identified clinically familiar as well as recently nominated metabolic markers that began to delineate a MELAS biochemical signature (Figure 2).

Untargeted metabolomics, combined with cheminformatics and analytical chemistry, revealed changes in 3 families of metabolites. To complement the targeted metabolomics approach we also analyzed all 134 plasma samples on an untargeted LC-MS platform (Massachusetts General Hospital) in full-scan mode, in which it was feasible for us to perform follow-up analytical chemistry on anonymous peaks. This platform detected levels for 5584 features, of which 237 could be putatively assigned a chemical identity (e.g., lactate, alanine, $\alpha$-hydroxybutyrate) based on a retention time library, while 5347 remained unidentified.

We prioritized the unidentified features based on their ability to discriminate patients with MELAS from controls after correcting for age, sex, and BMI. Applying a 2\% FDR cutoff, we found 536 total peaks were significant, of which only 31 were readily identified on the basis of retention time standards; 6 of these 31 (lactate, pyruvate, alanine, C2:0-carnitine, C3:0-carnitine, $\alpha$-hydroxybutyrate) were among the 23 significant metabolites identified on the targeted platform (Figure $3 \mathrm{~A}$, black dots). As the strongest peaks that distinguished cases from controls were many of the 505 unidentified features, we searched for potential compound matches using only their accurate exact masses in the Human Metabolome Database (HMDB) (54). As we manually reviewed the top 505 unidentified peaks, 3 metabolite families repeatedly emerged as candidate matches: N-lactoyl-amino acids, $\beta$-hydroxy acylcarnitines (BOHCAs), and $\beta$-hydroxy fatty acids (BOHFAs).

The first group of markers we explored in the unidentified peaks were 4 putative N-lactoyl-amino acids (Figure 3A, red dots). To our knowledge, these metabolites have only been identified twice previously in humans and have been proposed to be products of reverse proteolysis between lactate and amino acids (Figure 3B) $(55,56)$. Using LC-MS/MS, we confirmed that fragmentation of these 4 peaks matched authentic standards as well as previous reports of $4 \mathrm{~N}$-lactoyl-amino acids: N-lactoylphenylalanine (Figure 3C), N-lactoyl-leucine/isoleucine (unable to distinguish), N-lactoyl-tyrosine, and N-lactoyl-valine (55). Levels of these N-lactoyl-amino acids were significantly increased in patients with MELAS compared with controls (Figure 3D) and were strongly correlated to one another $\left(\mathrm{R}^{2}>0.8\right)$. We searched the LC-MS data for masses of all possible N-lactoyl-amino acids and found 7 putative additional members. However, only 3 (putative N-lactoyl-alanine, N-lactoyl-threonine, and N-lactoyl-gluta- 
A

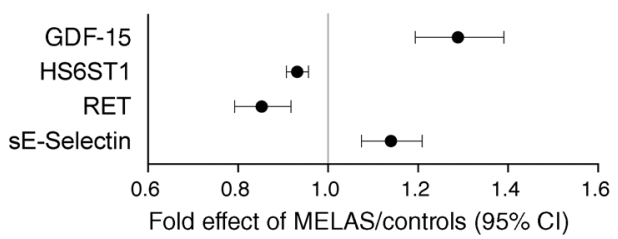

B

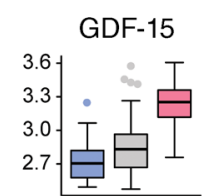

D

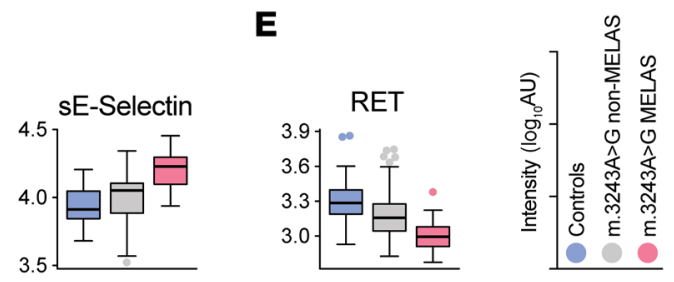

$\mathbf{F}$

C HS6ST1

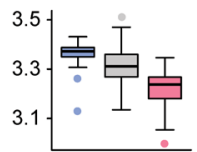

E

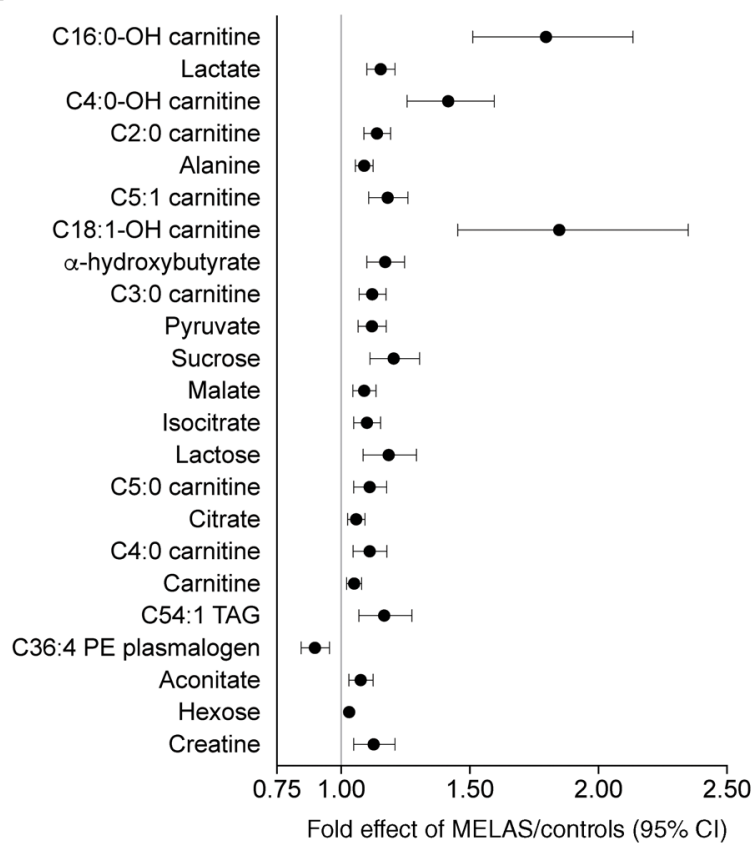

G

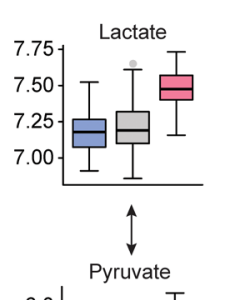

H

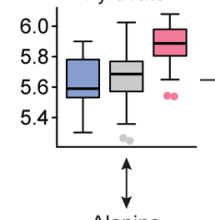

$\mathbf{H}$
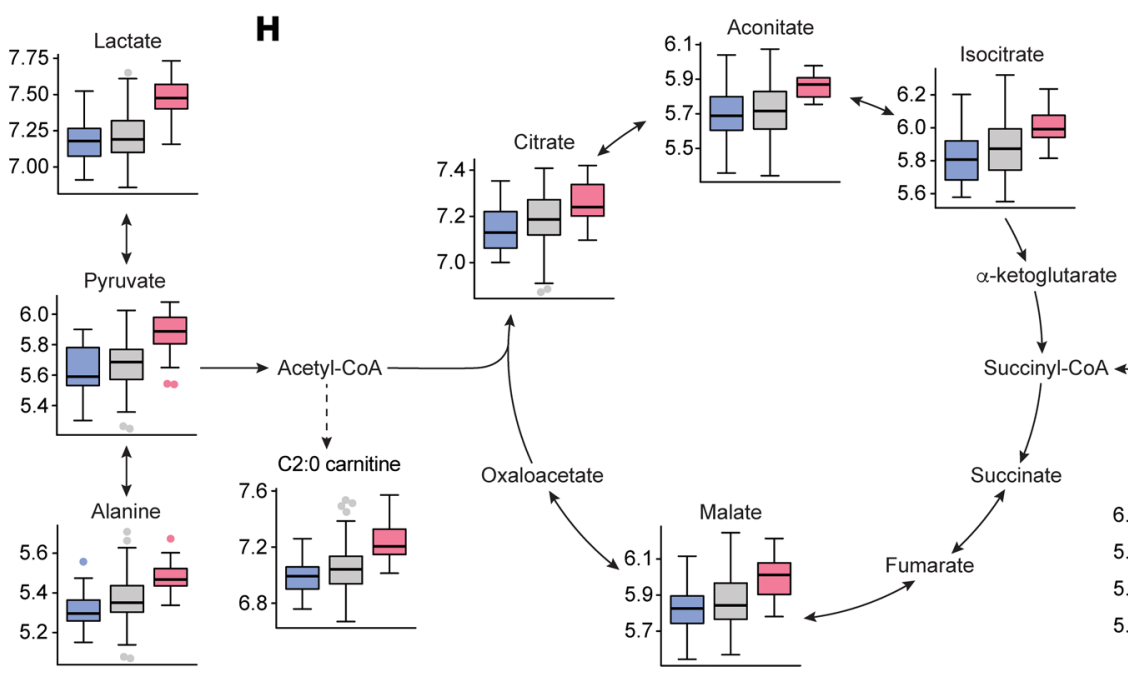

Oxaloacetate
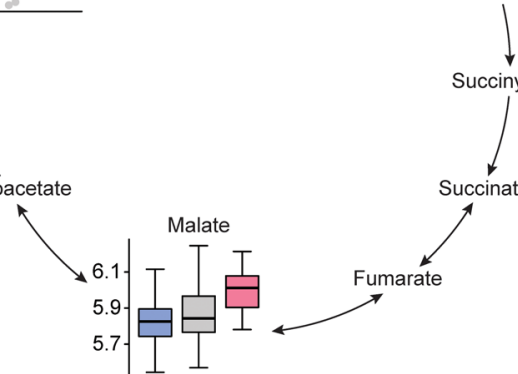

I $\alpha$-hydroxybutyrate

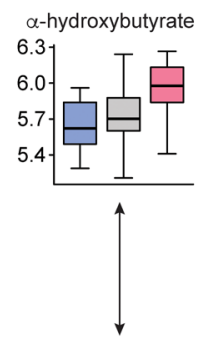

Succinyl-CoA «- - Propionyl-CoA $\longleftarrow \alpha$-ketobutyrate
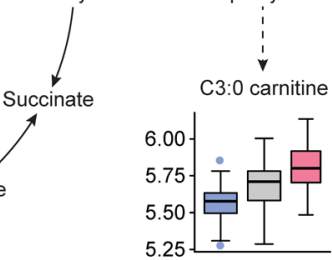

J
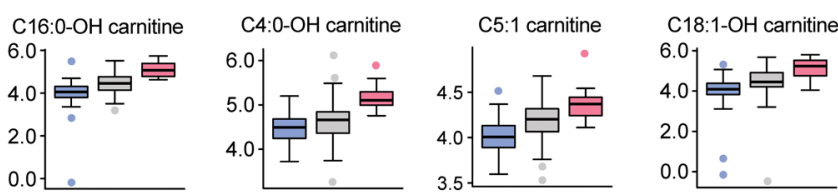

$\mathbf{L}$
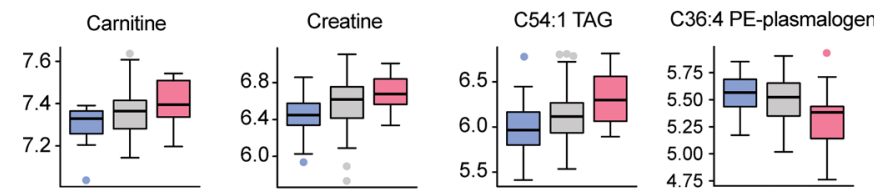

K

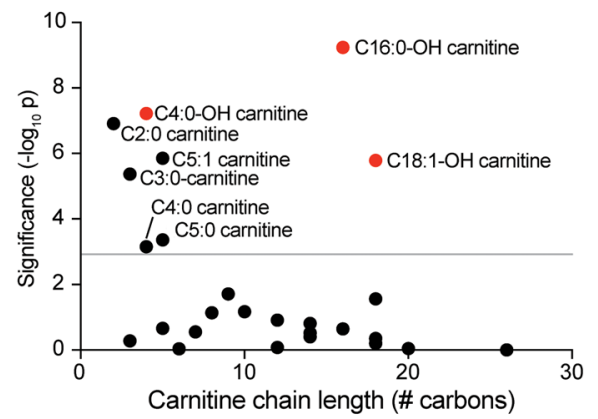


Figure 2. Comparison of $\mathbf{1 3 1 0}$ proteins and $\mathbf{3 7 6}$ targeted metabolites in plasma of patients with MELAS and controls. (A) Four proteins discriminate patients with MELAS $(n=16)$ from controls $(n=24)$ at the $2 \%$ FDR threshold with the indicated fold effects and $95 \%$ CI. Plots $(\mathbf{B}-\mathbf{E}, \mathbf{G}-\mathbf{J}$, and $\mathbf{L})$ show box (median with quartiles) and whisker (1.5 $\times$ IQR) plots of controls, m.3243A $>$ G non-MELAS and patients with MELAS for each analyte meeting the $2 \%$ FDR threshold with individual data points plotted only for outliers. All show $\log _{10}(\mathrm{AU})$ after correction for age, sex, BMI, and batch. (B) Growth differentiation factor 15 (GDF-15). (C) HS6ST1, heparan-sulfate 6-0-sulfotransferase 1. (D) sE-selectin, soluble E-selection. (E) RET proto-oncogene (RET). (F) Twenty-three metabolites from the targeted platform significantly discriminate patients with MELAS $(n=20)$ from controls $(n=32)$, with the indicated fold effects and $95 \%$ Cls. (G-J and L) Box and whisker plots for 18 of the top metabolites. (K) The significances shown as $-\log _{10}(P$ value) of each of the 26 different acylcarnitine species identified by the targeted metabolomics platform are shown according to carbon chain length with the gray line at $y=2.92$ marking the $2 \%$ FDR threshold. The $P$ values shown here are the results of a regression analysis controlling for age, sex, BMI, and batch (see Methods).

mine) were significantly correlated $\left(\mathrm{R}^{2}>0.5\right)$ to the 4 confirmed N-lactoyl-amino acids and only 2 (N-lactoyl-alanine and Nlactoyl-glutamine) met our 2\% FDR threshold, though neither were as substantially increased as the 4 confirmed species. While their biological role is unclear, these results nominate $4 \mathrm{~N}$ lactoyl-amino acids as potential MELAS markers.

The second metabolite family that emerged among the unidentified features consisted of 15 hydroxy-acylcarnitines ranging from 4 to 18 carbons in length (Figure $3 \mathrm{~A}$, cyan dots) and included 3 hydroxy-acylcarnitines highlighted by our targeted platform. To verify these hydroxy-acylcarnitines, we confirmed their retention times with 4 purchased standards (see Methods). Although we were unable to determine the exact position of the hydroxyl group, the $\beta$-position is consistent with prior observations in a diabetes mouse model (57). Moreover, the $\beta$-position is also consistent with inhibition of the $\mathrm{NAD}^{+}$-dependent dehydrogenation of $\beta$-hydroxyacyl-CoA species during fatty acid $\beta$-oxidation with elevated $\mathrm{NADH} / \mathrm{NAD}^{+}$ratio. The BOHCA family observed in the untargeted platform included some of the most significant of all features detected (Figure 3, A and E). This observation complemented the results from the targeted platform to more completely describe the MELAS acylcarnitine signature to include BOHCAs of all lengths as well as short acylcarnitines.

The third family spotlighted by the untargeted platform were 10 hydroxy-fatty acids of lengths ranging from 6 to 18 carbons (Figure 3A yellow dots, and Figure 3F). As noted above, we suspected the hydroxyl group is in the $\beta$-position as an elevated $\mathrm{NADH} / \mathrm{NAD}^{+}$ratio would interrupt fatty acid $\beta$-oxidation. We confirmed the retention times of 6 hydroxy-fatty acids using authentic standards. Using LC-MS/MS fragmentation of standards, we found the $\beta$-hydroxy fatty acid (and not the $\alpha$-hydroxy forms) produced a $59.0134 \mathrm{~m} / \mathrm{z}$ transition upon fragmentation (Figure 4, A-D). We used this transition and an LC-MS/MS method to measure relative levels of 16 different $\beta$-hydroxy fatty acids ranging from 4 to 18 carbons, 13 of which were significantly elevated in patients with MELAS compared with controls (Figure 4, $\mathrm{E}$ and $\mathrm{F}$ ). In addition to identifying a new class of potential MELAS biomarkers, these observations point to a shared biochemical origin underlying the metabolic perturbations in hydroxylated acylcarnitines and fatty acids.

Circulating markers correlated with measures of severity. To monitor patients clinically, it is desirable to identify biomarkers that reflect disease severity. The categorical analysis described so far highlights circulating markers that distinguish patients with MELAS from controls, but do not necessarily correlate quantitatively with disease severity across the entire cohort of patients with m.3243A>G. A small handful of measures and markers of disease severity have previously been reported for MELAS. The Karnofsky score (100 indicates no impairment, 30 severe disability, 0 is death), a holistic measure of functional status, has been shown to decline in patients with MELAS (36) and is often used in clinical trials (58-60). Previous studies have shown that MRS ventricular lactate, and to a lesser extent, urine heteroplasmy, are markers of MELAS disease severity (37). These 3 previously reported measures and markers tend to distinguish patients from controls in our categorical analysis (Table 1, Table 2).

To identify circulating plasma markers that best reflect severity and directly compare them with previously reported readouts of severity, we determined their correlations with urine heteroplasmy, MRS ventricular lactate, and Karnofsky score (Figure 5, A-C, respectively). We determined the Kendall rank correlation $(\tau)$ between each measure of severity and (a) all 1310 proteins measured on the proteomics platform and (b) all 658 identified metabolites (including N-lactoyl amino acids, BOHFAs, and BOHCAs). We also included direct comparisons of each measure of severity. As expected, we confirmed MRS ventricular lactate and urine heteroplasmy are strongly correlated with one another and anticorrelated with Karnofsky score (i.e., higher levels are associated with lower Karnofsky scores) (Figure 5, A-C). Encouragingly, in all 3 comparisons, we found the analytes identified as significantly altered in our categorical analyses (black dots in Figure 5, A-C) perform comparably or better than urine heteroplasmy, ventricular lactate, or Karnofsky score. Specifically, GDF-15, BOHFAs, BOHCAs, and N-lactoylamino acids are among the most strongly correlated with established measures and markers of severity. Surprisingly, 2 plasma analytes (GDF-15 and $\beta-\mathrm{OH}-\mathrm{C} 16: 0$ carnitine) were the most strongly correlated across all 3 measures of severity (Figure $5, \mathrm{~A}^{-} \mathrm{C}$ ). We note that the markers most strongly correlated with ventricular lactate are enriched for BOHFAs and BOHCAs, while N-lactoyl-amino acids are more strongly correlated with urine heteroplasmy.

Among the 3 comparisons, the Karnofsky score is most reflective of disease severity and the set of analytes most strongly correlated to it includes GDF-15 as well as a mix of BOHCAs, BOHFAs, and N-lactoyl-amino acids (Figure 5, C-D). The top candidate markers accounted for the 2 most correlated and 10 of the most anticorrelated analytes (Figure 5C). Furthermore, 7 of the N-lactoyl-amino acids (Figure 5C), BOHFAs, and BOHCAs had correlations to Karnofsky score that outperformed plasma lactate. The RET protein had the highest positive correlation with Karnofsky score (Figure 5D). In conclusion, correlation with any of 3 previously reported measures of disease severity homes in on the very same candidate markers that distinguish patients with MELAS from controls and nominates these as monitoring biomarkers of MELAS. 
A

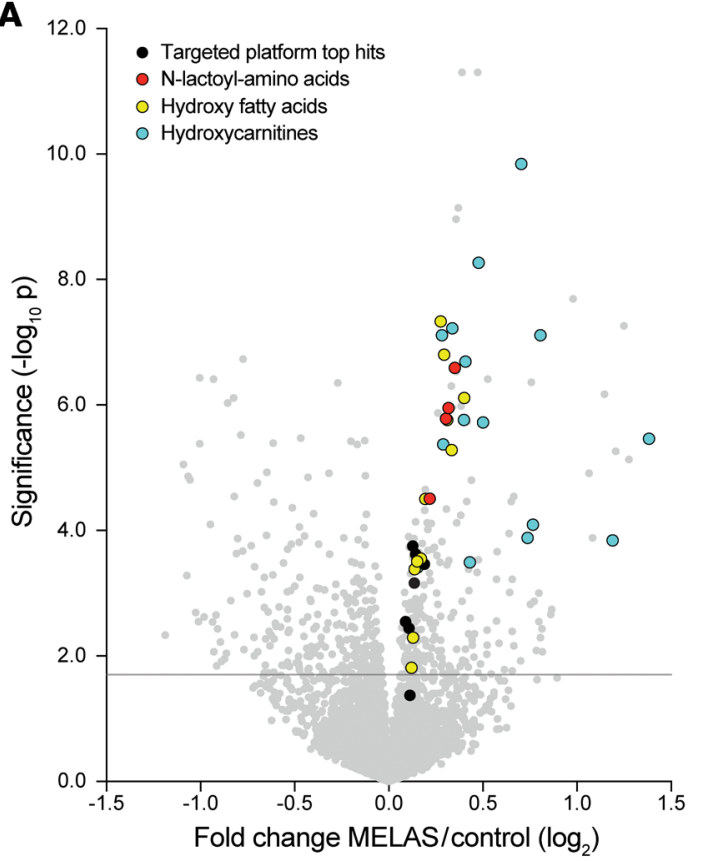

D
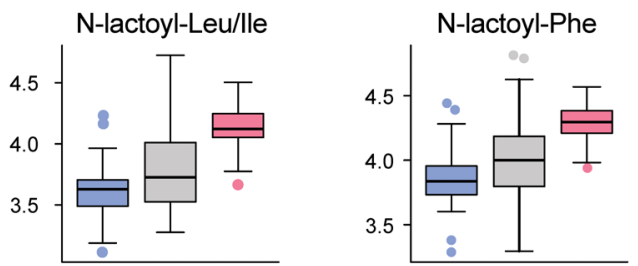

E

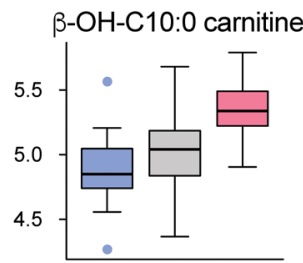

$\mathbf{F}$

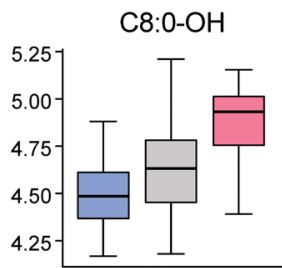

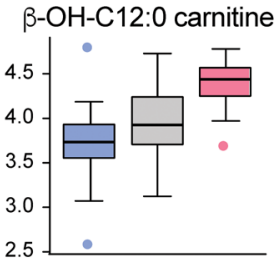

C10:0-OH

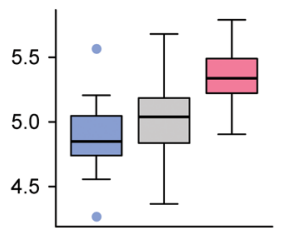

B

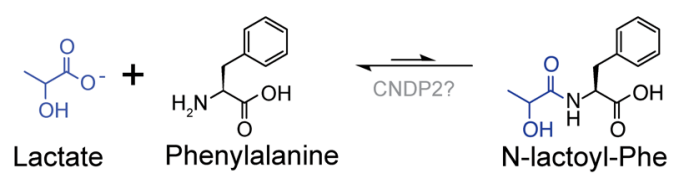

C

$\mathrm{N}$-lactoyl-Phe

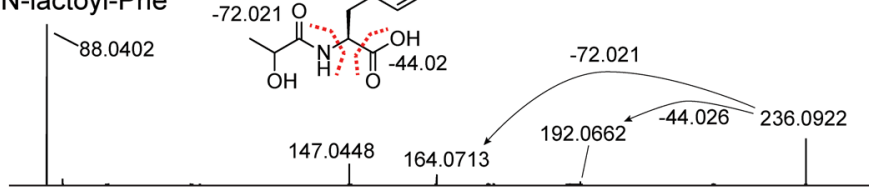

MELAS patient plasma

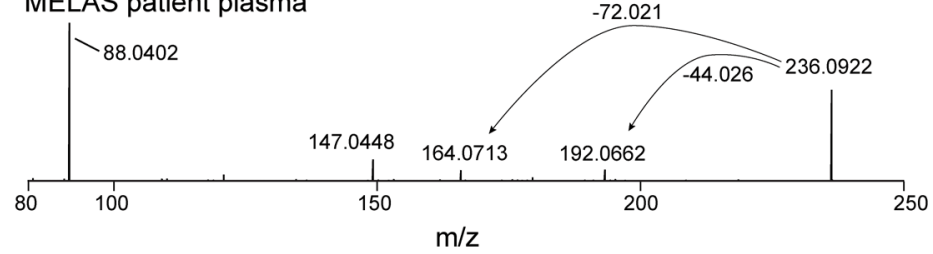

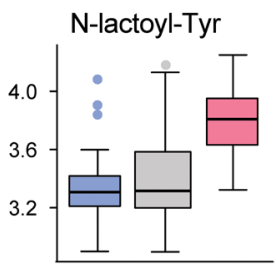

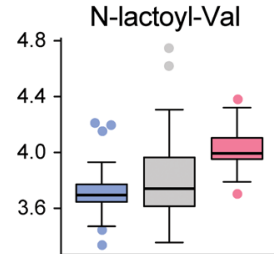

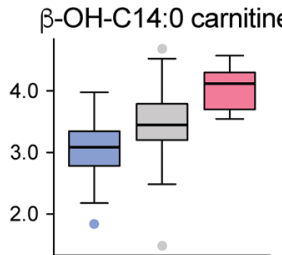

$\mathrm{C} 12: 0-\mathrm{OH}$

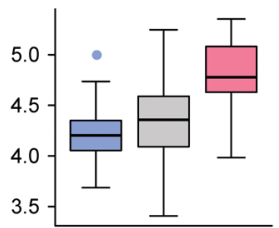

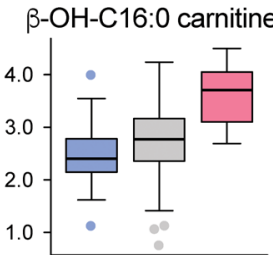

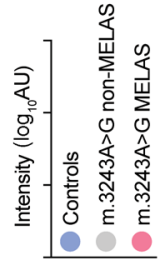

C14:0-OH

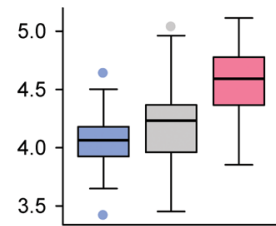

Figure 3. Biomarker discovery from untargeted metabolic profiling. (A) The untargeted metabolomics platform identified 5584 features. Each data point reflects the fold-change and $P$ value comparing patients with MELAS $(n=20)$ and controls $(n=32)$. Two hundred thirty-seven of the features were identified and 6 of those features (black dots) were identified by the targeted platform. Overall, 536 features met the 2\% FDR threshold indicated by a gray line at $y=1.7$. Three biochemical families appeared when searching for potential chemical matches by mass in HMDB: N-lactoyl-amino acids (red dots), hydroxy-fatty acids (yellow dots), and hydroxyacyl carnitines (cyan dots). (B) Four significant peaks matched N-lactoyl-amino acids. The origin of these metabolites is not known, though they have been proposed to be catalyzed as shown by reverse proteolysis. (C) A chemically synthesized N-lactoylphenylalanine standard has the same fragmentation pattern as the peak observed in a sample from a patient with MELAS. (D-F) Graphs following the formatting of Figure 2 show the distributions of (D) $4 \mathrm{~N}$-lactoyl-amino acids, (E) 4 of the 12 hydroxycarnitines, and (F) 4 of the 10 hydroxy-fatty acids in controls $(n=32)$ in m.3243A>C non-MELAS patients $(n=82)$ and patients with MELAS $(n=20)$. 

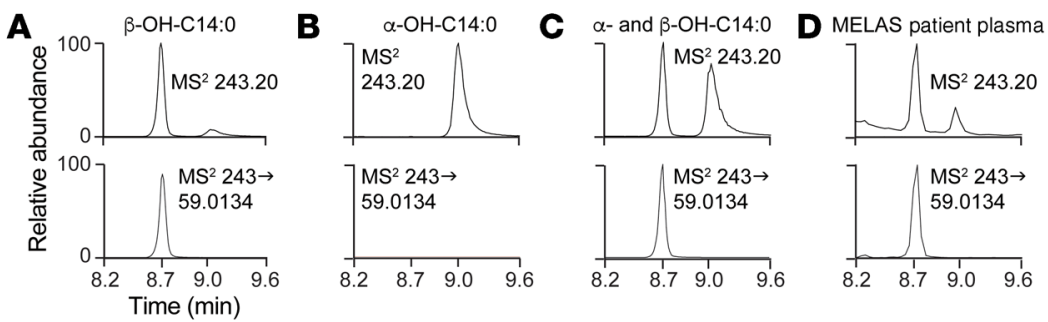

$\mathbf{E}$

\begin{tabular}{|c|c|}
\hline$\beta-\mathrm{OH}$ fatty acid & $P$ value \\
\hline$\beta-\mathrm{OH}$ C4:0 & 0.16 \\
\hline$\beta-\mathrm{OH}$ C6:0 & $4.6 \times 10^{-7}$ \\
\hline$\beta-\mathrm{OH}$ C8:0 & $8.4 \times 10^{9}$ \\
\hline$\beta-\mathrm{OH} \mathrm{C10:0}$ & $3.6 \times 10^{-9}$ \\
\hline$\beta-\mathrm{OH}$ C10:1 & 0.54 \\
\hline$\beta-\mathrm{OH} C 12: 0$ & $2.8 \times 10^{-8}$ \\
\hline$\beta-\mathrm{OH}$ C12:1 & $2.4 \times 10^{-7}$ \\
\hline$\beta-\mathrm{OH} C 14: 0$ & $7.9 \times 10^{-8}$ \\
\hline$\beta-\mathrm{OH} \mathrm{C14:1}$ & $1.5 \times 10^{-7}$ \\
\hline$\beta-\mathrm{OH}$ C14:2 & $3.2 \times 10^{-5}$ \\
\hline$\beta-\mathrm{OH} C 16: 0$ & $4.1 \times 10^{-7}$ \\
\hline$\beta-\mathrm{OH}$ C16:1 & $7.7 \times 10^{-9}$ \\
\hline$\beta-\mathrm{OH}$ C16:2 & $7.4 \times 10^{-8}$ \\
\hline$\beta-\mathrm{OH}$ C18:0 & 0.50 \\
\hline$\beta-\mathrm{OH}$ C18:1 & $1.7 \times 10^{-8}$ \\
\hline$\beta-\mathrm{OH}$ C18:2 & $5.7 \times 10^{-9}$ \\
\hline
\end{tabular}
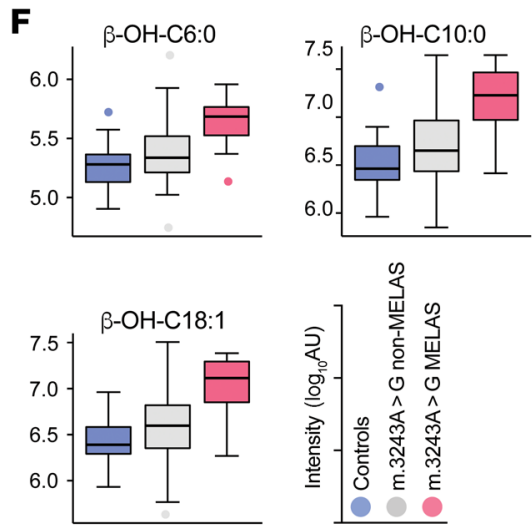

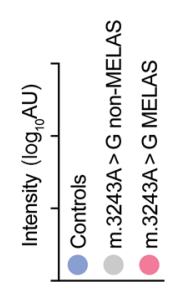

Figure 4. BOHFAs are significantly increased in MELAS

(A) Fragmentation of a standard of $\beta-\mathrm{OH}-\mathrm{C} 14: 0$ produces a $59.0134 \mathrm{~m} / \mathrm{z}$ fragment ion but (B) fragmentation of $\alpha-\mathrm{OH}-\mathrm{C} 14: 0$ does not. (C) A mixture of $\alpha$ - and $\beta-\mathrm{OH}-\mathrm{C} 14: 0$ standards can be separated based on retention time and the $59.0134 \mathrm{~m} / \mathrm{z}$ fragment ion. Each BOHFA standard tested produced a $59.0134 \mathrm{~m} / \mathrm{z}$ fragment ion (data not shown). (D) We quantified the relative level of $\beta-\mathrm{OH}-\mathrm{C} 14: 0$ in each sample using the exact mass and the 59.0134 $\mathrm{m} / \mathrm{z}$ transition; the extracted ion chromatogram for 1 representative patient with MELAS is shown. (E) Sixteen BOHFAs were quantified with their respective masses and the $59.0134 \mathrm{~m} / \mathrm{z}$ transition. The table shows the Wilcoxon rank-sum $P$ value comparing 20 patients with MELAS and 32 controls. (F) Distributions of 3 representative BOHFAs, following the format in Figure 2.
Lability of identified metabolites in response to exercise. A limitation of the classic mitochondrial disease marker plasma lactate is its acute responsiveness to exertion that contributes to its limited specificity (61). To assess the lability of the top metabolite markers to exertion, we turned to a previously published cohort of 12 controls and 21 mitochondrial myopathy patients 3 of whom harbor the m.3243A $>$ G mutation (Supplemental Figure $3 \mathrm{~A}$ and ref. 61). Each individual performed an exercise cycling protocol during which blood was collected at rest, at maximal exertion, and 10 minutes after completion (Supplemental Figure 3B). Using an earlier iteration of our untargeted metabolomics platform we measured concentrations of lactate, $\alpha$-hydroxybutyrate, and relative levels of 6 of our most distinguishing metabolites. We were unable to obtain BOHCA measurements or resolve the position of hydroxyl groups of hydroxy fatty acids from this platform.

As expected, lactate was elevated in patients with mitochondrial myopathy and rose with exercise (Supplemental Figure $3 \mathrm{C})$. The $\alpha$-hydroxybutyrate concentration was also elevated in the patients with mitochondrial myopathy who were at rest but, unlike lactate, did not rise during exercise (Supplemental Figure 3D). Aconitate and creatine similarly showed significantly elevated levels at rest and were relatively stable through exercise (Supplemental Figure 3, E and F). N-lactoyl-leucine/isoleucine and N-lactoyl-valine were both substantially increased at rest and their relative levels increased slightly during exercise, consistent with prior observations (Supplemental Figure 3, G and H) (55). Finally, 2 hydroxy-fatty acids were significantly elevated in this mitochondrial myopathy cohort and did not rise with exercise (Supplemental Figure 3, I and J). Together, these measurements demonstrate a subset of our top metabolite markers extend to mitochondrial myopathy and are relatively stable during exertion.

Correlation among markers identified in the discovery analysis. An important question is whether the markers we have identified reflect independent underlying biochemical processes, or whether they are reflective of changes in a small number of core pathways. An ideal biomarker panel would have some redundancy while also spanning multiple core pathways.

We explored the correlation structure among some of the top scoring analytes. We considered the 100 samples for which both proteomics and metabolomics (Supplemental Table 1) data were available and computed Kendall rank correlations $(\tau)$ among 38 of the most discriminating analytes. This analysis included all 4 proteins, 23 targeted metabolites, 4 confirmed N-lactoyl-amino acids, 4 representative BOHFAs spanning 8 to 14 carbons, and 3 representative BOHCAs beyond the 3 identified by the targeted platform.

The analysis yields 3 prominent clusters (Figure 6A). One cluster consisted primarily of metabolites participating in cytosolic reactions, including lactate, pyruvate, and alanine, as well as the $\mathrm{N}$-lactoyl-amino acids, which were highly correlated to one another, likely reflecting their shared enzymatic origin. Unexpectedly, GDF-15 was connected to the same group. The largest cluster was dominated by intramitochondrial metabolites and included the BOHFAs, BOHCAs, and 4 TCA cycle intermediates (Figure $6 \mathrm{~A}$ ). The third subgroup consisted of the short acylcarnitines (C3:0-carnitine to C5:0-carnitine) and carnitine itself. Some of the top analytes do not show strong correlation to other analytes, likely reflecting their smaller effect sizes (Figure 2, A and F).

Quantitation and validation of prioritized metabolites in an independent m.3243A>G cohort. We next sought to validate a prioritized subset of the newly discovered metabolites in a completely independent cohort, now using stable-isotope dilution methods to quantify plasma concentrations of metabolites of interest. We selected 22 metabolites for validation based on 4 criteria: (a) sampling of metabolites across correlation groups (Figure 6A), (b) inclusion of classical markers as positive controls, (c) inclusion of potentially novel markers, and (d) technical feasibility (Figure 6A, circles adjacent to names). We 
A

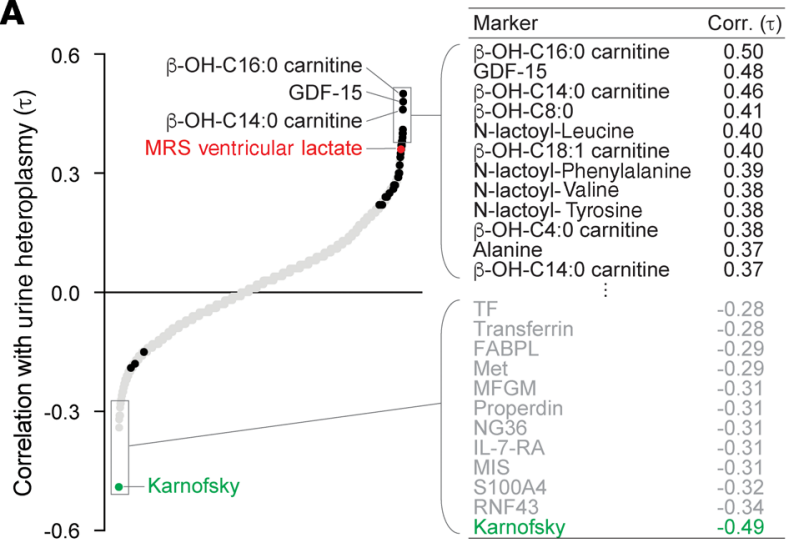

C

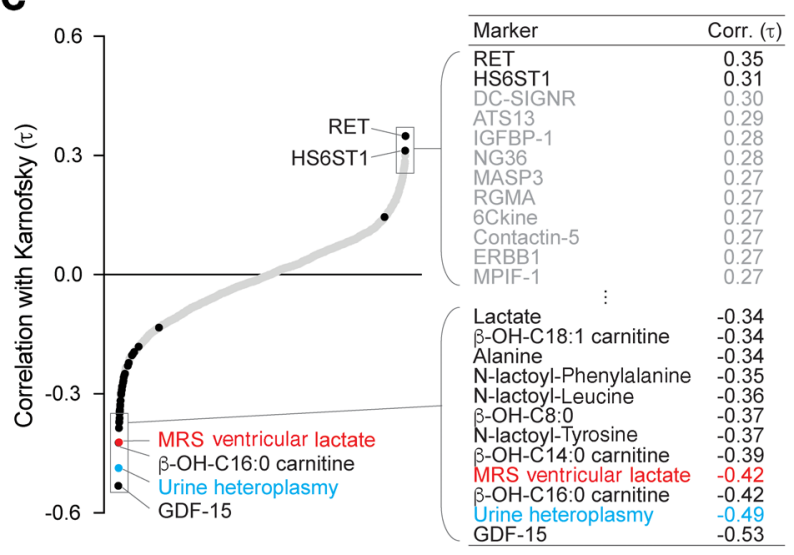

B

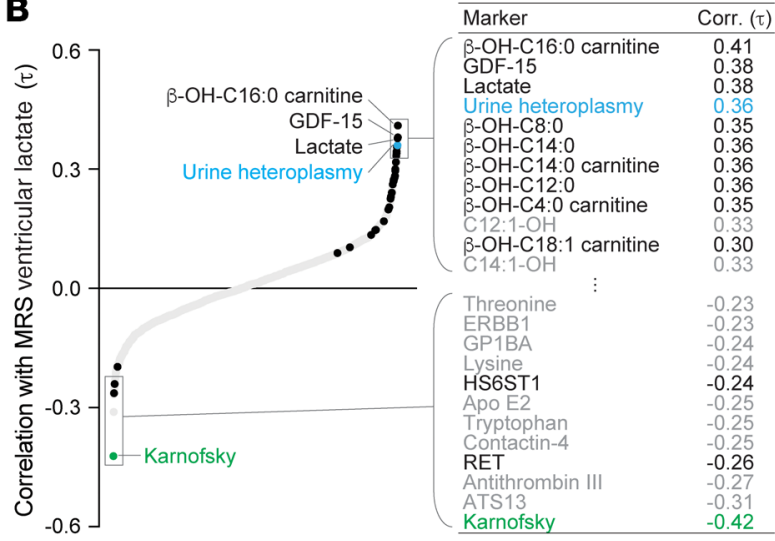

D Ventricular lactate
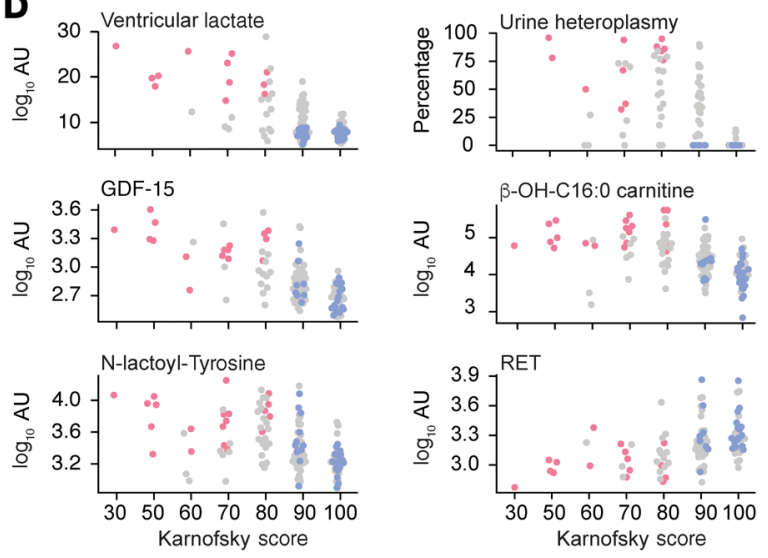

Control m.3243A>G non-MELAS m.3243A>G MELAS

Figure 5. Correlation of plasma markers, brain lactate, or urine heteroplasmy with measures of disease severity. Kendall rank correlation coefficient (Corr. $(\tau))$ of all 1978 proteins and identified metabolites from targeted and untargeted platforms with 3 measures of severity: (A) urine heteroplasmy, (B) MRS ventricular lactate, and (C) Karnofsky score. MRS ventricular lactate is highlighted in red, urine heteroplasmy in cyan, and Karnofsky score in green. Identified analytes found to be significant from the proteomics, targeted metabolomics, and untargeted metabolomics platforms are represented as black dots and the remainder as gray dots. The 12 most correlated and anticorrelated analytes are listed with those identified as candidate markers shown in black text and the remaining in gray text. (D) Distributions of Karnofsky scores for select analytes in controls, m.3243A>C non-MELAS patients and patients with MELAS. One data point for $\beta-\mathrm{OH}-\mathrm{C16:0}$ carnitine has been excluded as it was unmeasured in a control.

developed 2 stable-isotope dilution LC-MS methods to measure plasma concentrations of the 22 metabolites in an independently collected validation cohort consisting of 32 individuals: 7 m.3243A $>$ G patients with MELAS, 9 m.3243A $>$ G non-MELAS patients, and 16 controls matched for age, sex, and BMI (Figure 6B and Supplemental Table 2). We note that the male-tofemale ratio of patients with MELAS was balanced in the validation cohort, whereas it was skewed to females in the discovery cohort. We also determined concentrations for 20 patients with MELAS and 32 controls in the discovery cohort. While these methods are not clinically approved, the measured concentrations were comparable to values produced by clinical assays or reported in prior publications $(55,62,63)$.

Of the 22 metabolites quantified, 20 remained significantly increased $(P<0.05)$ in the discovery cohort and 19 of these were also significantly elevated in the validation cohort (Figure $6 \mathrm{C}$ ). In addition to classic markers like lactate and pyruvate, we found that $\alpha$-hydroxybutyrate, malate, and creatine, as well as $\mathrm{C} 2: 0$-carnitine and C3:0-carnitine, validated in both cohorts (Figure 6, C and D, Supplemental Figure 4, A, C-D). Among the metabolites iden- tified by the untargeted platform, all 3 BOHCAs, all 3 BOHFAs, and all $4 \mathrm{~N}$-lactoyl-amino acids replicated in the validation cohort (Figure 6, C and D, Supplemental Figure 4, E-G). Reflecting their correlation with severity, many of these markers also distinguish patients with MELAS from the m.3243A $>$ G non-MELAS patients.

In summary, 19 of the 22 assayed metabolites were significantly elevated in the validation cohort with the most discriminating being BOHCAs, BOHFAs, N-lactoyl amino acids, and alanine (Figure 6C). Combined with their correlation to severity and the stability of many to exertion, this validated set of 19 metabolites and 1 protein (GDF-15) comprise a MELAS clinical panel.

\section{Discussion}

The m.3243A $>\mathrm{G}$ mutation is the most common genetic cause of heteroplasmic mtDNA disease, the most famous of which is MELAS. These disorders are emblematic of the manifold challenges we face with mitochondrial disease. While molecular diagnoses of mitochondrial disease can be readily secured through genetic testing, the lack of validated biomarkers has hindered clinical management and rigorous testing of novel therapies. To 
A

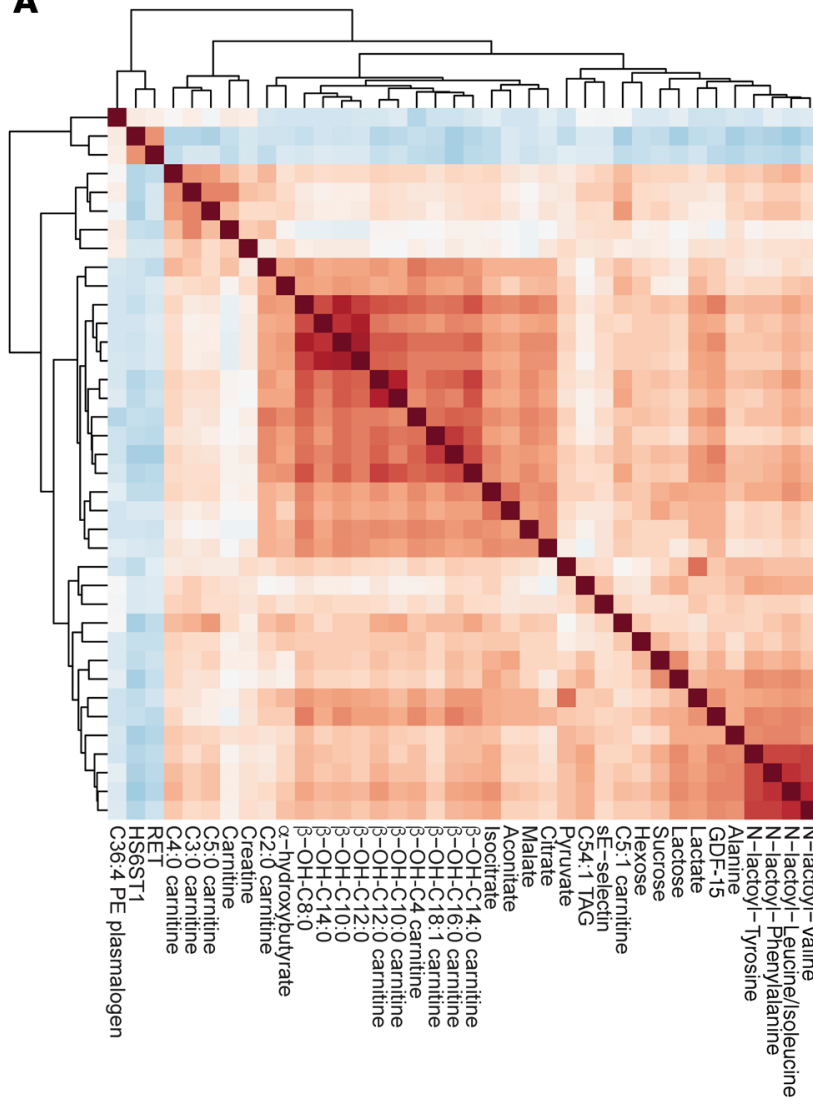

B

\begin{tabular}{lcc}
\hline & \multicolumn{2}{c}{ Validation cohort } \\
\cline { 2 - 3 } & MELAS & Controls \\
\hline $\mathrm{N}$ & 7 & 16 \\
Sex, male:female & $4: 3$ & $6: 10$ \\
Age (years), mean (range) & $37.2(21-60)$ & $31.1(23-55)$ \\
BMI, mean (range) & $18.3(14.5-21.5)$ & $22.8(18.6-27.2)$ \\
\hline
\end{tabular}

C

\begin{tabular}{|c|c|c|}
\hline & $\begin{array}{l}\text { Discovery } \\
\text { cohort }(P)\end{array}$ & $\begin{array}{l}\text { Validation } \\
\text { cohort }(P)\end{array}$ \\
\hline$\beta-\mathrm{OH}-\mathrm{C} 14: 0$ carnitine & $8.70 \times 10^{-8}$ & $4.08 \times 10^{-6}$ \\
\hline \multirow{2}{*}{$\begin{array}{l}\beta-\mathrm{OH}-\mathrm{C} 12: 0 \text { carnitine } \\
\beta-\mathrm{OH}-\mathrm{C} 8: 0\end{array}$} & $1.20 \times 10^{-7}$ & $8.16 \times 10^{-6}$ \\
\hline & $1.50 \times 10^{-7}$ & $2.86 \times 10^{-5}$ \\
\hline \multirow{2}{*}{$\begin{array}{l}\mathrm{N} \text {-lactoyl-Phenylalanine } \\
\text { Alanine }\end{array}$} & $2.40 \times 10^{-7}$ & $7.75 \times 10^{-5}$ \\
\hline & $2.40 \times 10^{-7}$ & $2.69 \times 10^{-4}$ \\
\hline $\mathrm{N}$-lactoyl-Tyrosine & $5.10 \times 10^{-7}$ & $2.69 \times 10^{-4}$ \\
\hline \multirow{2}{*}{$\begin{array}{l}\mathrm{N} \text {-lactoyl-Valine } \\
\beta-\mathrm{OH}-\mathrm{C} 16: 0 \text { carnitine }\end{array}$} & $5.40 \times 10^{-7}$ & $8.16 \times 10^{-6}$ \\
\hline & $8.5 \times 10^{-7}$ & $1.31 \times 10^{-4}$ \\
\hline & $8.70 \times 10^{-7}$ & $1.63 \times 10^{-5}$ \\
\hline \multirow{3}{*}{$\begin{array}{l}\beta-\mathrm{OH}-\mathrm{C} 12: 0 \\
\mathrm{~N} \text {-lactoyl-Leucine/Isoleucine } \\
\text { Lactate }\end{array}$} & $1.20 \times 10^{-6}$ & $1.63 \times 10^{-5}$ \\
\hline & $1.30 \times 10^{-6}$ & $8.16 \times 10^{-6}$ \\
\hline & $1.30 \times 10^{-6}$ & $4.08 \times 10^{-6}$ \\
\hline \multirow{2}{*}{$\begin{array}{l}\text { C2:0 carnitine } \\
\alpha \text {-hydroxybutyrate }\end{array}$} & $1.50 \times 10^{-6}$ & $1.22 \times 10^{-4}$ \\
\hline & $2.40 \times 10^{-5}$ & $7.38 \times 10^{-4}$ \\
\hline Malate & $2.60 \times 10^{-5}$ & $9.18 \times 10^{-3}$ \\
\hline Pyruvate & $5.00 \times 10^{-5}$ & $4.89 \times 10^{-5}$ \\
\hline \multirow{2}{*}{$\begin{array}{l}\text { Creatine } \\
\text { C3.0 carnitine }\end{array}$} & $2.20 \times 10^{-4}$ & $1.64 \times 10^{-2}$ \\
\hline & $3.70 \times 10^{-3}$ & $4.70 \times 10^{-4}$ \\
\hline C4:0 carnitine & $9.40 \times 10^{-3}$ & $2.97 \times 10^{-3}$ \\
\hline Carnitine & 0.019 & 0.051 \\
\hline \multirow{2}{*}{$\begin{array}{l}\text { Glucose } \\
\text { C5:0 carnitine }\end{array}$} & 0.092 & 0.111 \\
\hline & 0.18 & 0.088 \\
\hline
\end{tabular}
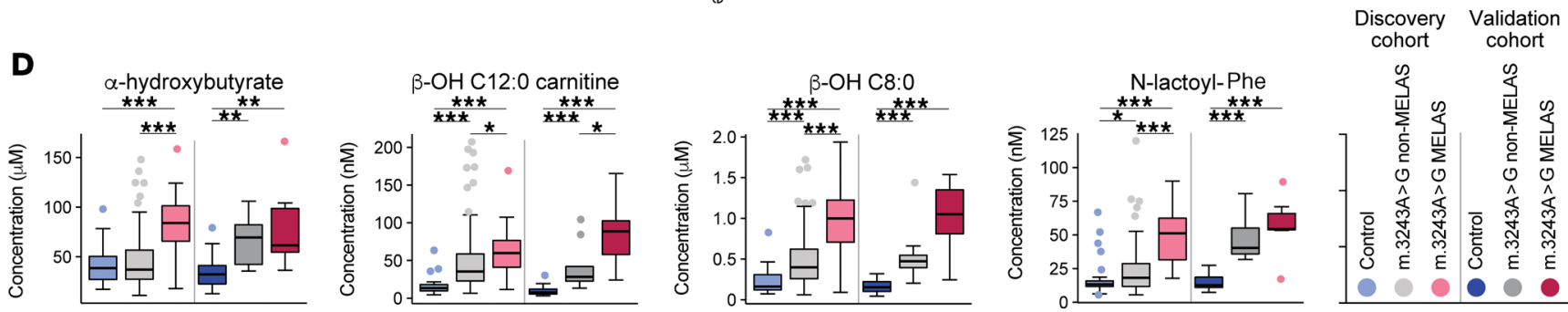

Figure 6. Correlations among most discriminating analytes and validation of top metabolites. (A) Proteomic profiling combined with targeted and untargeted metabolomic profiling revealed 4 proteins and 34 metabolites that significantly discriminate patients with MELAS from controls. The heatmap displays Kendall rank correlation coefficients $(\tau)$ among these 38 analytes over 16 patients with MELAS, 60 m.3243A>C non-MELAS patients, and 24 controls (set in which both proteomics and metabolomics was performed, Supplemental Table 1) and reveals several groups within which there is high correlation. Twenty-two metabolites from across the correlational groups were chosen for validation and are indicated by circles; filled circles were significantly $(P<0.05)$ different in the validation cohort. (B) We collected plasma samples from a separate validation cohort of 16 controls and 16 patients with the m.3243A $>$ G variant (7 of whom had MELAS) seen at 2 institutions (Supplemental Table 3). (C) Using 2 LC-MS methods, absolute concentrations of 22 metabolites were measured in the discovery (MELAS and controls only) and validation cohorts. The Wilcoxon rank-sum test was used to compare the MELAS and controls in each cohort, and 19 of the 22 metabolites validated with a $P$ value less than 0.05 . Metabolites are ordered by their discovery cohort $P$ value. (D) Distributions for 4 representative metabolites in discovery and validation cohorts following the format of Figure 2 with significance as indicated: ${ }^{*} P<0.05,{ }^{* *} P<0.01$, and ${ }^{* *} P<0.001$. The Wilcoxon rank-sum test was used to compare metabolite levels within each cohort.

systematically search for disease biomarkers that also reflect severity our study design incorporated a combination of deep phenotypic characterization and application of targeted proteomics and metabolomics as well as untargeted, full-scan metabolomics to discovery and validation cohorts. We have validated a suite of 20 plasma biomarkers that include markers previously used in classic diagnostic algorithms (e.g., lactate, alanine, pyruvate, acylcarnitines) (Figure 1). In addition to validating recently reported candidate markers (GDF-15, $\alpha$-hydroxybutyrate), our untargeted platform followed by exact mass cheminformatics and in-depth analytical chemistry revealed N-lactoyl-amino acids, BOHFAs, and BOHCAs as new families of m.3243A $>$ G markers that are among the strongest to date. Our integrated phenotypic, radiographic, proteomic and metabolomic characterization of the discovery cohort enabled us to discover that several of the plasma markers including GDF-15 strongly correlate with disease severity, performing as well as MRS ventricular lactate. These markers also provide key insights into biochemical pathogenesis.

Elevated NADH/NAD was the major determinant of the plasma metabolic signature of MELAS and non-MELAS m.3243A>G 


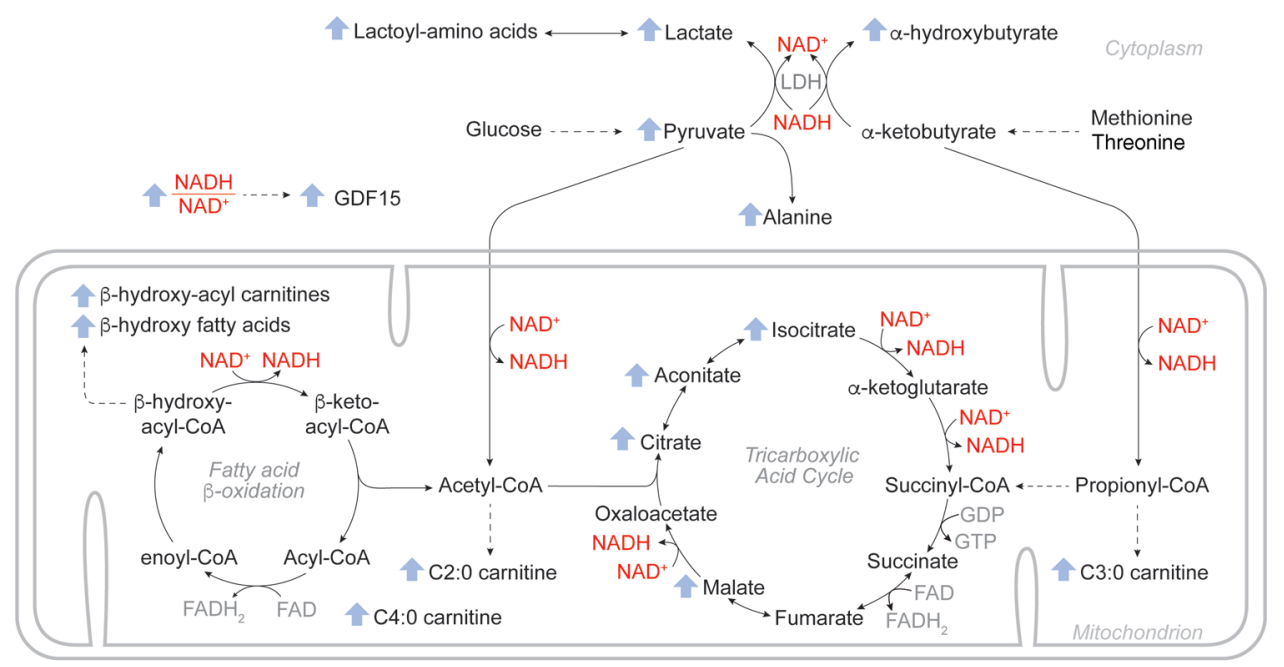

Figure 7. NADH-reductive stress drives the metabolic signature in MELAS. Depicting the primary analytes emerging from our targeted and untargeted platforms on their respective metabolic pathways in cytoplasm and within mitochondria revealed that biochemical reactions sensitive to the $\mathrm{NADH} /$ $N A D^{+}$ratio strongly impacted the plasma metabolite content in MELAS. disease. In principle, there are many downstream consequences of interrupting the respiratory chain: ATP deficiency, spillage of ROS, rewiring of one-carbon intermediates, or elevation of $\mathrm{NADH} / \mathrm{NAD}^{+}$(termed NADH-reductive stress). When the analytes from the 3 prominent clusters (Figure 6A) were projected on their respective metabolic pathways, the dominant theme of NADH-reductive stress was patently clear. We noted substrate accumulation at steps utilizing $\mathrm{NAD}^{+}$in 3 primary central metabolic avenues: LDH-related reactions, fatty acid $\beta$-oxidation, and the TCA cycle (Figure 7). NADH-reductive stress impacted 2 LDH-catalyzed reactions. First, the classic cytosolic redox pair, lactate and pyruvate, as well as the lactate/pyruvate ratio were significantly increased in both the discovery and validation cohorts (Supplemental Figure 4A). Accumulation of lactate likely drives the increased synthesis of N-lactoyl-amino acids by an unclear mechanism. Although reverse proteolysis via the cytosolic enzyme CNDP2 has been proposed (55), we noted that this is thermodynamically unfavorable and further studies are required to determine the in vivo provenance of these metabolites. Second, LDH catalyzed the reduction of $\alpha$-ketobutyrate to $\alpha$-hydroxybutyrate, accumulation of which has been demonstrated to reflect cytosolic NADH-reductive stress (64). The circulating metabolic signature of MELAS thus reflects NADH-reductive stress in both the cytoplasmic and mitochondrial compartments involving multiple pathways and builds on the classical observation of high $\mathrm{NADH} / \mathrm{NAD}^{+}$in the cytosol.

The BOHFAs and BOHCAs were the 2 strongest metabolite marker families emerging from our study and both were revealed through mining untargeted metabolomics data and point toward an inhibition of fatty acid $\beta$-oxidation in MELAS due to NADHreductive stress. To our knowledge, no specific pattern of acylcarnitine elevations are characteristic for MELAS, and our results indicated that the pattern consists of short-chain acylcarnitines (C2-, C3-, and C4-carnitine) and BOHCAs ranging from 4 to 18 carbons in length. Similarly, our results clarified the TCA cycle profile of MELAS to include intermediates preceding $\mathrm{NAD}^{+}$dependent steps. While our platforms did not reveal changes in $\beta$-hydroxybutyrate and acetoacetate (linked by $\beta$-hydroxybutyrate dehydrogenase) to be significant, we determined concentrations of these classic markers in the discovery and validation cohorts. $\beta$-hydroxybutyrate and the $\beta$-hydroxybutyrate/acetoacetate ratio were also significantly increased in both cohorts (Supplemental Figure 4B). These observations in combination indicate the pronounced role of mitochondrial NADH-reductive stress in shaping the circulating metabolic signature of MELAS.

At present, whether the redox imbalance in NADH-coupled metabolites is simply a marker of underlying disease or whether it formally contributes to organ pathology is not known. Addressing this question is important as it has critical therapeutic implications. It is notable that several experimental therapies aim to target $\mathrm{NADH} / \mathrm{NAD}^{+}$imbalance through manipulation of circulating metabolite levels using pyruvate supplementation or an engineered enzyme $(65,66)$. The metabolites identified here may be useful in monitoring the effects of these interventions.

GDF-15, a marker of the integrated stress response, is correlated with disease severity. Previous studies have nominated GDF-15 as a circulating biomarker of mitochondrial disease $(44,46,67)$, and in the current study, not only have we been able to validate this result, but we have been able to demonstrate that it is perhaps the strongest correlate of disease severity (Figure 5D). A key question is whether the reductive stress signature (reflected by NADHcoupled metabolites) is mechanistically related to the integrated stress response (reflected by high GDF-15). We recently demonstrated in cultured muscle cells that high $\mathrm{NADH} / \mathrm{NAD}^{+}$reductive stress is, in fact, a major driver of the activation of the integrated stress response to complex I deficiency in cultured myoblasts (68). Moreover, pyruvate therapy, which alleviates $\mathrm{NADH} / \mathrm{NAD}^{+}$ reductive stress, is also known to reduce GDF-15 in humans (67). Collectively, these previous studies, combined with our current metabolomics and proteomics, propose a model in which $\mathrm{NADH}$-reductive stress is likely the proximal biochemical defect in respiratory chain disease that then leads to the activation of the integrated stress response (Figure 7).

Other metabolic processes in the MELAS biochemical signature. While metabolites linked to $\mathrm{NAD}^{+}$-dependent reactions are clearly overrepresented in our top analytes, other biochemical mechanisms are most certainly at play too. For example, elevated plasma creatine may reflect conversion of phosphocreatine to defend ATP 
levels (53). Consistent with this explanation, phosphocreatine levels were modestly decreased (0.94-fold) and just missed our threshold for significance (adjusted $P=0.05$; Supplemental Figure 2B). We also noted metabolites in the anaplerotic pathway leading from propionyl-CoA to the TCA cycle (Supplemental Figure 2A) showed discordant changes: patients with MELAS showed a significant accumulation of C3-carnitine but a decrease in succinate, just missing our FDR threshold (adjusted $P=0.05$ ), while the intermediate, methylmalonate (MMA), was not significantly different. We hypothesize that this pattern is consistent with inhibition of the propionyl-CoA carboxylase reaction (Supplemental Figure 2A) and is supported by prior studies of this pathway, though the specific mechanism remains unclear (69).

MELAS markers were reflective more broadly of mitochondrial dysfunction. The signature of the NADH-reductive stress we observed in MELAS is likely a shared feature of many mitochondrial disorders of the respiratory chain. We found that patients with mitochondrial myopathy (Supplemental Figure 2) as well as patients with Leigh Syndrome French Canadian (LSFC, Supplemental Figure 5, and ref. 49), both of which have genetic defects in the respiratory chain, have overlapping metabolic changes. Eleven of the 31 plasma metabolites discriminating patients with LSFC from controls are also highlighted in our study, including classic markers as well as $\alpha$-hydroxybutyrate, 2 TCA cycle intermediates, 2 short chain acylcarnitines, and creatine (Supplemental Figure 5). Additionally, succinate was significantly decreased in patients with LSFC as well as in the MELAS discovery cohort but missed our 2\% FDR threshold (Supplemental Figure 2A). While the metabolic changes in LSFC and MELAS are not identical, the overlapping signatures likely reflect a shared, common biochemical basis. Several of the metabolites identified here, including $\alpha$-hydroxybutyrate, creatine, and TCA cycle intermediates have also been observed in multiple other mitochondrial diseases $(38,50,51,53)$. The 3 BOHCAs identified by our targeted platform were, in fact, found to be elevated in a mouse model of Ndufs 4 deficiency (70). Finally, isolated mitochondria treated with respiratory chain inhibitors are unable to oxidize fatty acids and accumulate BOHFAs (71, 72). These observations indicate that NADH-reductive stress triggers a set of biochemically conserved alterations, aspects of which may be shared among respiratory chain disorders.

The biochemical changes we observe in MELAS may have an even broader purview beyond respiratory chain diseases. In fact, $\alpha$-hydroxybutyrate was first identified as a marker for early insulin resistance and indicates progression of diabetes $(62,73,74)$. Elevations in BOHCAs were found in a mouse model of diabetes (75). Finally, circulating GDF-15 appears to be one of the strongest markers of the aging process itself (76-79).

Insights into organ system pathology. Our metabolite and protein profiles raise important new hypotheses about organ system pathology in MELAS. Gastrointestinal symptoms such as vomiting or pseudo-obstruction are prominent features and may have plausible connections to 2 of our top protein markers, GDF-15 and RET. GDF-15 induces an anorexic response and weight loss by its action in the brainstem, where its receptor is a heterodimer of GDNF family receptor alpha like (GFRAL) and RET (46, $68,80,81)$. RET levels are lower in MELAS which is curious, as loss-of-function mutations in this gene are associated with
Hirschsprung disease in which regional colonic aganglionosis manifests as bowel obstruction.

The pathophysiological mechanisms driving stroke-like episodes in MELAS remain poorly understood but are thought to be different from those underlying acute ischemic strokes. Consistent with this, metabolomic signatures of ischemic stroke (either in the acute or remote setting) are distinct from our observations (82). Two possible drivers of MELAS stroke-like episodes that have been invoked include focal seizure triggering local energy depletion and vasoconstriction due to nitric oxide deficiency (83). While the latter is based on observations of lower levels of arginine or citrulline $(38,84,85)$, we did not observe such differences in the discovery cohort (Supplemental Figure 2D).

Patients with the m.3243A $>\mathrm{G}$ variant often suffer substantial decompensation in the setting of intercurrent infections $(83,86-$ 89). Two clues regarding the potential role of immune dysregulation emerge from our measurements: patients with MELAS have a subclinical relative leukocytosis (Table 2), and sE-selectin, a soluble vascular endothelial adhesion molecule involved in leukocyte extravasation that correlates with sepsis severity, is markedly elevated in patients with MELAS (Figure 2, A and D) $(83,90,91)$.

Our profiling may provide insight into several endocrine defects in MELAS. Diabetes is a common feature of MELAS and it is notable that we see consistent elevations in $\alpha$-hydroxybutyrate and BOHCAs, both of which have been linked to diabetes and may point to NADH-reductive stress as a shared underlying driver $(73,92)$. As previously reported, we observe a deficiency of T3 but not total thyroxine (T4) in patients with MELAS, suggesting a subclinical sick-euthyroid state (Table 2) (40). Patients with MELAS can manifest hypogonadism, and it is notable that our patients with MELAS had a significant decrease in HS6ST1 (Figure 2, A and C), which has been implicated in an inherited form of hypogonadotropic hypogonadism $(93,94)$.

Circulating markers of disease severity. One of the major hurdles in the clinical management and drug approval for mitochondrial disorders such as MELAS is the lack of facile, quantitative biomarkers with which to monitor disease progression and severity. Natural history studies have demonstrated markedly heterogeneous, unpredictable courses, with Karnofsky scores in half of patients declining by 12 points per year but stable or improving in the other half $(34,36)$. The correlation of urine heteroplasmy with severity is confounded by the fact that heteroplasmy actually declines with age $(42,95)$. To date, the strongest quantitative biomarker of MELAS disease severity is ventricular lactate measured with brain NMR spectroscopy, available at specialized centers. Here, our top validated markers showed striking correlation with multiple measures of disease severity, including the Karnofsky score, and even outperformed ventricular lactate. We noted that the biomarker correlation to Karnofsky score depends on the phenotypic extreme of the MELAS cohort (Figure 5D). Future prospective studies will be required to determine whether the metabolite and protein markers we have identified track quantitatively with disease severity in other cohorts of patients with mitochondrial disease.

While the analytes validated here are likely to be clinically beneficial, future challenges lie in delineating the sets of markers that optimize sensitivity and specificity, report organ-specific 
pathology, and predict progression. We noted that the identified markers were modestly elevated (with statistical significance) in patients with MELAS when compared with controls, which may necessitate a combinatorial approach (as with other clinical panels) to enhance sensitivity, specificity, and utility. Additionally, our cohort size precluded meaningful correlations between analytes and specific disease features or supplement use, which may only become apparent with markedly larger cohorts. The identified metabolites appear to also be elevated in pediatric Leigh syndrome, raising the possibility that most of our markers are not private to MELAS but rather more broadly reflect impairment of the respiratory chain. We anticipate that larger, prospective studies of other genetic and phenotypic forms of mitochondrial disease will be a requisite step toward full clinical implementation.

In conclusion, application of proteomic and metabolomic profiling to discovery and validation cohorts allowed us to nominate and validate a set of 20 MELAS-monitoring biomarkers (Figure 1). In addition to classic markers (e.g., lactate and alanine), these analytes include recently identified markers (e.g., $\alpha$-hydroxybutyrate and GDF-15) and brand-new classes of markers such as N-lactoyl-amino acids, BOHFAs, and BOHCAs that we discovered and validated in human patients. Mechanistically, the new markers we have identified correlate with disease severity comparable to MRS ventricular lactate and also point to NADH-reductive stress as a key component of the biochemical pathogenesis of mitochondrial diseases, and the integrated stress response, which has recently been mechanistically linked to high $\mathrm{NADH} / \mathrm{NAD}^{+}$ratios. These biochemical insights, combined with the ability to track disease severity, should be helpful in the development of new treatment strategies for mitochondrial disorders.

\section{Methods}

Please see the supplemental material for detailed Methods.

Study approval. All samples and clinical information of the discovery cohort were collected following approval of a human studies protocol (IRB-AAAB1425) by the IRB at Columbia University Irving Medical Center. All samples for the validation cohort were collect- ed following approval of the IRB at Massachusetts General Hospital (2003P002032 and 2016P001517). The exercise plasma samples were collected following approval of the IRB at University of Texas Southwestern Medical Center (092010-077). All samples were processed for metabolomics and proteomics following approval of a secondary use of deidentified samples protocol (2010P000089) by the IRB at Massachusetts General Hospital.

\section{Author contributions}

VKM, DCDV, MH, DO, and RS designed the study. KE, ES, and RF coordinated discovery cohort activities. MAW and RS collected samples from the validation cohort. RGH collected samples from the exercise cohort. $\mathrm{CBC}$ and $\mathrm{KP}$ performed targeted metabolite profiling. RS and OSS performed untargeted metabolic profiling, confirmation of novel metabolites, and absolute quantification. BR performed statistical analyses. DCS and XM performed MRS measurements. AK synthesized isotopically labeled N-lactoyl-amino acids. RS and VKM assembled the manuscript.

\section{Acknowledgments}

Stephen Hersh, advisor to the Marriott family and foundation, had the vision to spearhead this unique collaborative effort and the team is deeply thankful for his guidance through each stage of this endeavor. We thank Jun Peng for early technical contributions. This work was supported by the Marriott Mitochondrial Disorders Collaborative Research Network (to VKM, RS, BR, OSS, MAW, MH, DO), National Institutes of Health (R01AR050597 to RGH, F32GM133047 to OSS, P01HD080642 to DCD).

Address correspondence to: Vamsi K. Mootha, 185 Cambridge Street, CPZN 6400, Boston, Massachusetts 02114 USA. Phone: 617.643.3059. Email: vamsi@hms.harvard.edu. Or to: Darryl C. De Vivo, 710 West 168th Street, Neurological Institute, Room 2-201, New York, New York 10032 USA. Phone: 212.305.5244; Email: dcd1@cumc.columbia.edu. Or to: Michio Hirano, 630 West 168th Street, P\&S 4-423, New York, New York 10032 USA. Phone: 1.212.305.1048; Email:mh29@columbia.edu.
1. MacMunn CA. Further observations on myohaematin and the histohaematins. JPhysiol. 1887;8(2):51-65.

2. Skladal D, et al. Minimum birth prevalence of mitochondrial respiratory chain disorders in children. Brain. 2003;126(8):1905-1912.

3. Gorman GS, et al. Prevalence of nuclear and mitochondrial DNA mutations related to adult mitochondrial disease. Ann Neurol. 2015;77(5):753-759.

4. Munnich A, et al. Clinical aspects of mitochondrial disorders. JInherit Metab Dis. 1992;15(4):448-455.

5. Fannin SW, et al. Aging selectively decreases oxidative capacity in rat heart interfibrillar mitochondria. Arch Biochem Biophys. 1999;372(2):399-407.

6. Tranah GJ, et al. Mitochondrial DNA m.3243A $>\mathrm{G}$ heteroplasmy affects multiple aging phenotypes and risk of mortality. Sci Rep. 2018;8(1):11887.

7. Trifunovic A, et al. Somatic mtDNA mutations cause aging phenotypes without affecting reac- tive oxygen species production. Proc Natl Acad Sci U S A. 2005;102(50):17993-17998.

8. Kujoth GC, et al. Mitochondrial DNA mutations, oxidative stress, and apoptosis in mammalian aging. Science. 2005;309(5733):481-484.

9. Patti ME, et al. Coordinated reduction of genes of oxidative metabolism in humans with insulin resistance and diabetes: potential role of PGC1 and NRF1. Proc Natl Acad Sci U S A. 2003;100(14):8466-8471.

10. El-Hattab AW, et al. MELAS GeneReviews [Internet]. University of Washington, Seattle; 1993-2020. February 27, 2001. Updated November 29, 2018. https://www.ncbi.nlm.nih. gov/books/NBK1233/.

11. Goto Y, et al. A mutation in the tRNA(Leu)(UUR) gene associated with the MELAS subgroup of mitochondrial encephalomyopathies. Nature. 1990;348(6302):651-653

12. Barca E, et al. Mitochondrial diseases in North America: an analysis of the NAMDC Registry. Neurol Genet. 2020;6(2):e402.
13. Pavlakis SG, et al. Mitochondrial myopathy, encephalopathy, lactic acidosis, and strokelike episodes: a distinctive clinical syndrome. Ann Neurol. 1984;16(4):481-488.

14. Kaufmann P, et al. Natural history of MELAS associated with mitochondrial DNA m.3243A $>$ G genotype. Neurology. 2012;77(22):1965-1971.

15. Elliott HR, et al. Pathogenic mitochondrial DNA mutations are common in the general population. Am J Hum Genet. 2008;83(2):254-260.

16. Manwaring $\mathrm{N}$, et al. Population prevalence of the MELAS A3243G mutation. Mitochondrion. 2007;7(3):230-233.

17. Majamaa K, et al. Epidemiology of A3243G, the mutation for mitochondrial encephalomyopathy, lactic acidosis, and strokelike episodes: prevalence of the mutation in an adult population. Am J Hum Genet. 1998;63(2):447-454.

18. Yatsuga S, et al. MELAS: a nationwide prospective cohort study of 96 patients in Japan. Biochim Biophys Acta. 2012;1820(5):619-624.

19. Hirano M, et al. Emerging therapies for 
mitochondrial diseases. Essays Biochem. 2018;62(3):467-481.

20. Sage-Schwaede A, et al. Exploring mTOR inhibition as treatment for mitochondrial disease. Ann Clin Transl Neurol. 2019;6(9):1877-1881.

21. Wortmann SB, et al. Whole exome sequencing of suspected mitochondrial patients in clinical practice. J Inherit Metab Dis. 2015;38(3):437-443.

22. Steele HE, et al. Monitoring clinical progression with mitochondrial disease biomarkers. Brain. 2017;140(10):2530-2540.

23. Kobayashi M, et al. Two cases of NADH-coenzyme Q reductase deficiency: relationship to MELAS syndrome. J Pediatr. 1987;110(2):223-227.

24. Hirano M, et al. Melas: an original case and clinical criteria for diagnosis. Neuromuscul Disord. 1992;2(2):125-135.

25. Xiao W, Loscalzo J. Metabolic responses to reductive stress. Antioxid Redox Signal. 2020;32(18):1330-1347.

26. Chance B, et al. Hydroperoxide metabo lism in mammalian organs. Physiol Rev. 1979;59(3):527-605.

27. Nishizawa M, et al. A mitochondrial encephalomyopathy with cardiomyopathy. A case revealing a defect of complex I in the respiratory chain. J Neurol Sci.1987;78(2):189-201.

28. Ichiki T, et al. Deficiency of subunits of Complex I and mitochondrial encephalomyopathy. Ann Neurol. 1988;23(3):287-294.

29. Williamson D, et al. The redox state of free nicotinamide-adenine dinucleotide in the cytoplasm and mitochondria of rat liver. Biochem J. 1967;103(2):514-527.

30. Suomalainen A. Biomarkers for mitochondrial respiratory chain disorders. JInherit Metab Dis. 2011;34(2):277-282.

31. Peron F, Caldwell BV. Steroid hydroxylations by guinea-pig adrenal tissue fractions. Biochim Biophys Acta. 1967;143(3):532-546.

32. Grady JP, et al. mtDNA heteroplasmy level and copy number indicate disease burden in m.3243A $>$ G mitochondrial disease. EMBO Mol Med. 2018;10(6):e8262.

33. Rahman S, et al. Decrease of $3243 \mathrm{~A} \rightarrow \mathrm{G}$ mtDNA mutation from blood in MELAS syndrome: a longitudinal study. Am J Hum Genet. 2001;68(1):238-240.

34. Mehrazin M, et al. Longitudinal changes of mtDNA A3243G mutation load and level of functioning in MELAS. Am JMed Genet A. 2009;149(4):584-587.

35. Sue CM, et al. Detection of MELAS A3243G point mutation in muscle, blood and hair follicles. J Neurol Sci.1998;161(1):36-39.

36. Kaufmann P, et al. Natural history of MELAS associated with mitochondrial DNA m.3243A $>\mathrm{G}$ genotype. Neurology. 2011;77(22):1965-1971.

37. Kaufmann P, et al. Cerebral lactic acidosis correlates with neurological impairment in MELAS. Neurology. 2004;62(8):1297-1302.

38. Buzkova J, et al. Metabolomes of mitochondrial diseases and inclusion body myositis patients: treatment targets and biomarkers. EMBO Mol Med. 2018;10(12):e9091.

39. Esterhuizen K, et al. A urinary biosignature for mitochondrial myopathy, encephalopathy, lactic acidosis and stroke like episodes (MELAS).
Mitochondrion. 2019;45:38-45.

40. Kaufmann P, et al. Protean phenotypic features of the A3243G mitochondrial DNA mutation. Arch Neurol. 2009;66(1):85-91.

41. Whittaker RG, et al. Urine heteroplasmy is the best predictor of clinical outcome in the m.3243A $>$ G mtDNA mutation. Neurology. 2009;72(6):568-569.

42. Fayssoil A, et al. Prediction of long-term prognosis by heteroplasmy levels of the m.3243A $>\mathrm{G}$ mutation in patients with the mitochondrial encephalomyopathy, lactic acidosis and stroke-like episodes syndrome. Eur J Neurol. 2017;24(2):255-261.

43. Chow J, et al. Mitochondrial disease and endocrine dysfunction. Nat Rev Endocrinol. 2017;13(2):92-104.

44. Montero R, et al. GDF-15 is elevated in children with mitochondrial diseases and is induced by mitochondrial dysfunction. PLoS One. 2016;11(2):1-15.

45. Yatsuga S, et al. Growth differentiation factor 15 as a useful biomarker for mitochondrial disorders. Ann Neurol. 2015;78(5):814-823.

46. Chung HK, et al. Growth differentiation factor 15 is a myomitokine governing systemic energy homeostasis. JCell Biol. 2017;216(1):149-165.

47. Bernier FP, et al. Diagnostic criteria for respiratory chain disorders in adults and children. Neurology. 2002;59(9):1406-1411.

48. Morava E, et al. Mitochondrial disease criteria: diagnostic applications in children. Neurology. 2006;67(10):1823-1826

49. Thompson Legault J, et al. A metabolic signature of mitochondrial dysfunction revealed through a monogenic form of Leigh syndrome. Cell Rep. 2015;13(5):981-989.

50. Jain IH, et al. Hypoxia as a therapy for mitochondrial disease. Science. 2016;352(6281):54-61.

51. Semeraro M, et al. The urinary organic acids profile in single large-scale mitochondrial DNA deletion disorders. Clin Chim Acta. 2018;481:156-160.

52. Goodman RP, et al. Hepatic reductive stress is a causal determinant of common variation in metabolic traits. Nature. 2020;583(7814):122-126.

53. Shaham O, et al. A plasma signature of human mitochondrial disease revealed through metabolic profiling of spent media from cultured muscle cells. Proc Natl Acad Sci U S A. 2010;107(4):1571-1575.

54. Wishart DS, et al. HMDB 4.0: the human metabolome database for 2018. Nucleic Acids Res. 2018;46(D1):D608-D617.

55. Jansen RS, et al. N-lactoyl-amino acids are ubiquitous metabolites that originate from CNDP2-mediated reverse proteolysis of lactate and amino acids. Proc Natl Acad Sci US A. 2015;112(21):6601-6606.

56. Hagenfeldt L, Naglo AS. New conjugated urinary metabolites in intermediate type maple syrup urine disease. Clin Chim Acta. 1987;169(1):77-83.

57. Su X, et al. Accumulation of long-chain acylcarnitine and 3-hydroxy acylcarnitine molecular species in diabetic myocardium: identification of alterations in mitochondrial fatty acid processing in diabetic myocardium by shotgun lipidomics. Biochemistry. 2005;44(13):5234-5245.

58. Patil CG, et al. Whole brain radiation therapy
(WBRT) alone versus WBRT and radiosurgery for the treatment of brain metastases. Cochrane Database Syst Rev. 2017;9(9):CD006121.

59. Reyes-Botero G, et al. Temozolomide plus bevacizumab in elderly patients with newly diagnosed glioblastoma and poor performance status: an ANOCEF phase II trial (ATAG). Oncologist. 2018;23(5):524-e44.

60. Salehifar E, et al. Efficacy and safety of bupropion in cancer-related fatigue, a randomized double blind placebo controlled clinical trial. $B M C$ Cancer. 2020;20(1):1-9.

61. Delaney NF, et al. Metabolic profiles of exercise in patients with McArdle disease or mitochondrial myopathy. Proc Natl Acad Sci. 2017;114(31):8402-8407.

62. Tricò D, et al. Elevated a-hydroxybutyrate and branched-chain amino acid levels predict deterioration of glycemic control in adolescents. JClin Endocrinol Metab. 2017;102(7):2473-2481.

63. Costa CG, et al. Simultaneous analysis of plasma free fatty acids and their 3-hydroxy analogs in fatty acid $ß$-oxidation disorders. Clin Chem. 1998;44(3):463-471.

64. Goodman RP, et al. Hepatic reductive stress is a causal determinant of common variation in metabolic traits. Nature. 2020;583(7814):122-126.

65. Fujii T, et al. Efficacy of pyruvate therapy in patients with mitochondrial disease: a semi-quantitative clinical evaluation study. Mol Genet Metab. 2014;112(2):133-138.

66. Patgiri A, et al. An engineered enzyme that targets circulating lactate to alleviate intracellular NADH:NAD ${ }^{+}$imbalance. Nat Biotechnol. 2020;38(3):309-313.

67. Fujita Y, et al. GDF15 is a novel biomarker to evaluate efficacy of pyruvate therapy for mitochondrial diseases. Mitochondrion. 2015;20:34-42.

68. Mick E, et al. Distinct mitochondrial defects trigger the integrated stress response depending on the metabolic state of the cell. Elife. 2020;9:e49178.

69. Bui D, et al. The effect of 2-ketobutyrate on mitochondrial substrate-level phosphorylation. Neurochem Res. 2019;44(10):2301-2306.

70. Leong DW, et al. Proteomic and metabolomic analyses of mitochondrial complex I-deficient mouse model generated by spontaneous B2 short interspersed nuclear element (SINE) insertion into NADH dehydrogenase (ubiquinone) Fe-S protein 4 (Ndufs4) gene. J Biol Chem. 2012;287(24):20652-20663.

71. Moore $\mathrm{KH}$, et al. Incomplete fatty acid oxidation by ischemic heart: beta-hydroxy fatty acid production. Am J Physiol. 1982;14(2):451-H265.

72. Jin SJ, et al. Incomplete fatty acid oxidation. The production and epimerization of 3-hydroxy fatty acids. J Biol Chem. 1992;267(1):119-125.

73. Gall WE, et al. $\alpha$-hydroxybutyrate is an early biomarker of insulin resistance and glucose intolerance in a nondiabetic population. PLOS One. 2010;5(5):e10883.

74. Varvel SA, et al. Serum $\alpha$-hydroxybutyrate $(\alpha-\mathrm{HB})$ predicts elevated $1 \mathrm{~h}$ glucose levels and early-phase $\beta$-cell dysfunction during OGTT. BMJ Open Diabetes Res Care. 2014;2(1):e000038.

75. Kuo TH, et al. Defective oxidative metabolism of heart mitochondria from genetically diabetic 
mice. Diabetes. 1983;32(9):781-787.

76. Tanaka $\mathrm{T}$, et al. Plasma proteomic signature of age in healthy humans. Aging Cell. 2018;17(5):e12799.

77. Welsh JB, et al. Analysis of gene expression identifies candidate markers and pharmacological targets in prostate cancer. Cancer Res. 2001;61(16):5974-5978.

78. Wang T, et al. GDF15 is a heart-derived hormone that regulates body growth. EMBO Mol Med. 2017;9(8):1150-1164.

79. Semba RD, et al. Elevated plasma growth and differentiation factor 15 is associated with slower gait speed and lower physical performance in healthy community-dwelling adults. J Gerontol $A$ Biol Sci Med Sci. 2020;75(1):175-180.

80. Metallo CM, et al. Evaluation of $13 \mathrm{C}$ isotopic tracers for metabolic flux analysis in mammalian cells. J Biotechnol. 2009;144(3):167-174.

81. Mullican SE, et al. GFRAL is the receptor for GDF15 and the ligand promotes weight loss in mice and nonhuman primates. Nat Med. 2017;23(10):1150-1157.

82. Ke C, et al. Metabolomics facilitates the discovery of metabolic biomarkers and pathways for ischemic stroke: a systematic review. Metabolomics.
2019;15(12):152.

83. Fryer RH, et al. Mitochondrial encephalomyopathy lactic acidosis and stroke-like episodes (MELAS): a case report and critical reappraisal of treatment options. Pediatr Neurol. 2016;56:59-61.

84. Koga Y, et al. L-arginine improves the symptoms of strokelike episodes in MELAS. Neurology. 2005;64(4):710-712.

85. El-Hattab AW, et al. Restoration of impaired nitric oxide production in MELAS syndrome with citrulline and arginine supplementation. $\mathrm{Mol}$ Genet Metab. 2012;105(4):607-614.

86. Sofou K, et al. A multicenter study on Leigh syndrome: disease course and predictors of survival. Orphanet J Rare Dis. 2014;9:52.

87. Edmonds JL, et al. The otolaryngological manifestations of mitochondrial disease and the risk of neurodegeneration with infection. Arch Otolaryngol Head Neck Surg. 2002;128(4):355-362.

88. Eom S, et al. Cause of death in children with mitochondrial diseases. Pediatr Neurol. 2017;66:82-88.

89. Walker MA, et al. Predisposition to infection and SIRS in mitochondrial disorders: 8 years' experience in an academic center. J Allergy Clin Immunol Pract. 2014;2(4):465-468.
90. Walker MA, et al. Powering the immune system: mitochondria in immune function and deficiency. JImmunol Res. 2014;2014:164309.

91. Kjaergaard AG, et al. The use of the soluble adhesion molecules sE-selectin, sICAM-1, sVCAM-1, sPECAM-1 and their ligands CD11a and CD49d as diagnostic and prognostic biomarkers in septic and critically ill non-septic ICU patients. APMIS. 2016;124(10):846-855.

92. Gao XH, et al. Quantitative H2S-mediated protein sulfhydration reveals metabolic reprogramming during the integrated stress response. Elife. 2015;4:e10067.

93. Howard SR, et al. HS6ST1 insufficiency causes self-limited delayed puberty in contrast with other GnRH deficiency genes. J Clin Endocrinol Metab. 2018;103(9):3420-3429.

94. Tornberg J, et al. Heparan sulfate 6-Osulfotransferase 1, a gene involved in extracellular sugar modifications, is mutated in patients with idiopathic hypogonadotrophic hypogonadism. Proc Natl Acad Sci U S A. 2011;108(28):11524-11529.

95. de Laat $P$, et al. Clinical features and heteroplasmy in blood, urine and saliva in 34 Dutch families carrying the m.3243A $>$ G mutation. 